\title{
Acidentes cirúrgicos na colecistectomia por laparotomia
}

Dissertação apresentada à Faculdade de Medicina da Universidade de São Paulo para obtenção do título de Mestre em Ciências.

Área de Concentração: Cirurgia do Aparelho Digestivo

Orientadora: Dra. Sônia Penteado 


\section{FICHA CATALOGRÁFICA}

Preparada pela Biblioteca da Faculdade de Medicina da Universidade de São Paulo

๑reprodução autorizada pelo autor

Neves, Carlos da Costa

Acidentes cirúrgicos na colecistectomia por laparotomia / Carlos da Costa Neves. -- São Paulo, 2003.

Dissertação(mestrado)--Faculdade de Medicina da Universidade de São Paulo. Departamento de Gastroenterologia.

Área de concentração: Cirurgia do Aparelho Digestivo.

Orientadora: Sônia Penteado.

Descritores: 1.LAPARATOMIA/estatística \& dados numéricos 2.COLECESTECTOMIA/métodos 3.DOENÇA IATROGÊNICA 4.VIAS BILIARES/cirurgia 5.COLANGIOGRAFIA/métodos 6.COLESTASIA INTRAHEPÁTICA/enzimologia 7.SEGUIMENTOS 8.GOIÁS (GO)

USP/FM/SBD-317/03 
Dedico este trabalho à minha esposa Selma, aos meus filhos Sandra e Carlos Gustavo, aos meus netos Juliana e Edison. 


\section{AGRADECIMENTOS}

À Dra. Sônia Penteado que, com segurança, me garantiu esta jornada.

Aos Professores Doutores

Bruno Zilberstein

Cláudio Bresciani

Ivan Ceconello

Manoel Maria

que, em momentos especiais, se fizeram estímulo, apoio, sabedoria e grandeza.

Destaco, também,

Dr. Luiz Arantes

Dr. Carlos Gustavo C L Neves

Acadêmico Frederico Dib e Castro

por suas atenções de força, sensibilidade e companheirismo.

A cada um,

obrigado. 


\section{SUMÁRIO}

Lista de abreviaturas e símbolos

Lista de tabelas

Lista de figuras

Lista de anexos

Resumo

Abstract

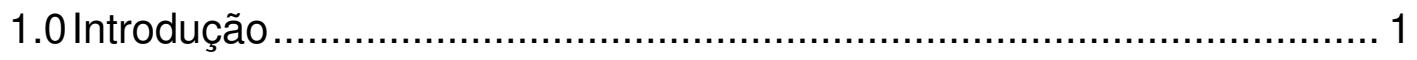

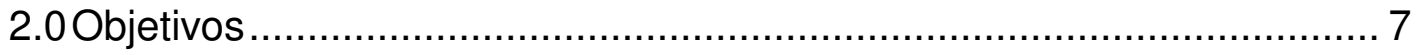

3.0 Casuística e Métodos ............................................................. 9

3.1 Casuística ....................................................................... 10

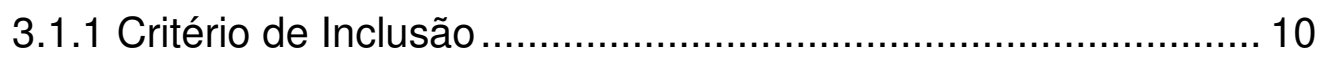

3.2 Método .................................................................... 12

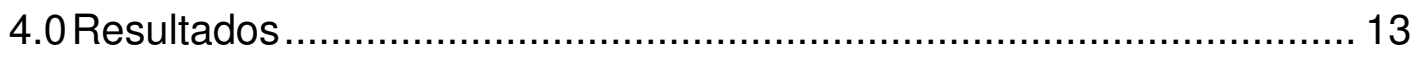

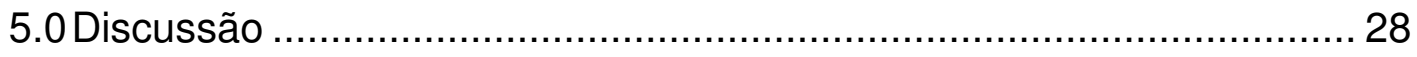

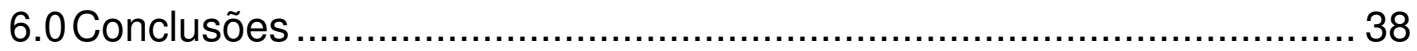

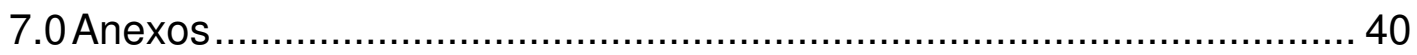

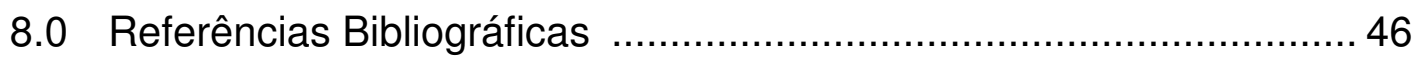




\section{LISTA DE ABREVIATURAS E SÍMBOLOS}

\begin{tabular}{|c|c|}
\hline $\mathrm{ClO}$ & Colangiografia Intra-Operatória \\
\hline CPER & Colangiopancreatografia Endoscópica Retrógrada \\
\hline CRM & Colangio-ressonância Magnética \\
\hline $\mathrm{CTPH}$ & Colangiografia Transparietohepática \\
\hline$C D$ & Coledocoduodenoanastomose \\
\hline DCD & Ducto Colédoco \\
\hline DHCo & Ducto Hepático Comum \\
\hline DHD & Ducto Hepático Direito \\
\hline DHE & Ducto Hepático Esquerdo \\
\hline DP & Desvio Padrão \\
\hline DVB & Drenagem da Via Biliar \\
\hline HC-UFG & Hospital das Clínicas da Universidade Federal de Goiás \\
\hline HJ & Hepaticojejunoanastomose \\
\hline LVB & Lesão de Via Biliar \\
\hline LVBP & Lesão da Via Biliar Principal \\
\hline $\mathrm{n}$ & Número de Pacientes \\
\hline$\%$ & Percentagem \\
\hline $\mathrm{RV}$ & Recusa Voluntária \\
\hline SA & Strasberg A \\
\hline SB & Strasberg B \\
\hline SC & Strasberg C \\
\hline SD & Strasberg D \\
\hline $\mathrm{SE}_{1}$ & Strasberg $E_{1}$ \\
\hline $\mathrm{SE}_{2}$ & Strasberg $\mathrm{E}_{2}$ \\
\hline $\mathrm{SE}_{3}$ & Strasberg $E_{3}$ \\
\hline $\mathrm{SE}_{4}$ & Strasberg $\mathrm{E}_{4}$ \\
\hline $\mathrm{Tc} A b$ & Tomografia Computadorizada de Abdômen \\
\hline USG Ab & Ultra-Sonografia de Abdômen \\
\hline VB & Vias Biliares \\
\hline VBP & Via Biliar Principal \\
\hline
\end{tabular}




\section{LISTA DE TABELAS}

Página

Tabela 1 - Número de pacientes operados de 1990 a 2002 .................. 14

Tabela 2 - Incidência de lesão relacionada à indicação da

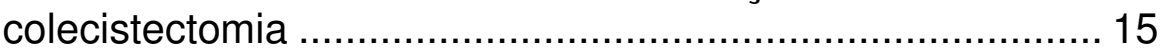

Tabela 3 - Incidência de lesão quanto aos sexos ................................ 15

Tabela 4 - Incidência de lesão relacionada à indicação da colecistectomia em outras regiões …………………….... 16

Tabela 5 - Índice de lesão quanto aos sexos ………………………... 16

Tabela 6 - Distribuição dos pacientes por faixa etária............................ 17

Tabela 7 - Tipo de incisão utilizada na colecistectomia e caráter da

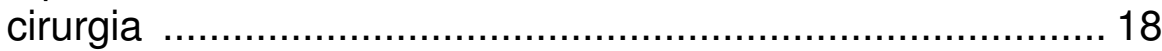

Tabela 8 - Sinais e sintomas dos pacientes pós-colecistectomia …...... 19

Tabela 9 - Pacientes submetidos a colangiografia ................................ 20

Tabela 10 - Classificação das lesões segundo Strasberg ……………...... 21

Tabela 11 - Ocasião do diagnóstico e tratamento da lesão da via biliar por colecistectomia . ........................................................... 22

Tabela 12 - Tratamento cirúrgico das lesões da via biliar ........................ 22

Tabela 13 - Tratamento por meio de drenagem da via biliar ................... 23

Tabela 14 - Tempo de permanência dos drenos ……………………... 23

Tabela 15 - Complicações pós-operatórias imediatas. Óbitos ................. 24

Tabela 16 - Mortalidade em relação à ocasião do tratamento ………………..24

Tabela 17 - Doenças associadas à admissão para tratamento cirúrgico

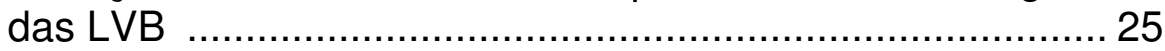

Tabela 18 - Evolução tardia. Lesão parcial .......................................... 25

Tabela 19 - Evolução tardia. Lesão total ................................................. 26

Tabela 20 - Complicações imediatas ………………………………... 27

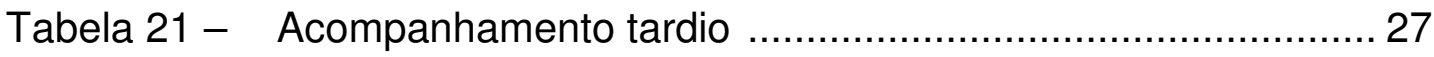




\section{LISTA DE FIGURAS}

Página

Figura 1 - Número de pacientes operados de 1990 a 2002 ..................... 14

Figura 2 - Distribuição dos pacientes quanto ao sexo .......................... 15

Figura 3 - Distribuição dos pacientes quanto ao sexo …...................... 16

Figura 4 - Distribuição dos pacientes por faixa etária........................... 17

Figura 5 - Tipo de incisão utilizada na colecistectomia e caráter da

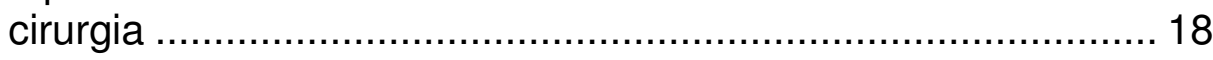

Figura 6 - Sinais e sintomas dos pacientes pós-colecistectomia .............. 19

Figura 7 - Pacientes submetidos a colangiografia ............................... 20

Figura 8 - Classificação das LVB segundo Strasberg .............................. 21

Figura 9 - Classificação das LVB segundo Strasberg ........................ 21

Figura 10 - Tratamento cirúrgico das LVB e o tipo de lesão ..................... 22

Figura 11 - Drenagem da via biliar .............................................. 23 


\section{LISTA DE ANEXOS}

Página

ANEXO 1 - Achados dos exames complementares dos pacientes com LVB tipo B e D segundo a classificação de Strasberg

ANEXO 2 - Achados dos exames complementares dos pacientes com LVB tipo E segundo a classificação de Strasberg.

ANEXO 3 - Intervalo entre a colecistectomia e as cirurgias corretivas nos pacientes que necessitaram de 2 procedimentos

ANEXO 4 - Intervalo entre a colecistectomia e as cirurgias corretivas nos pacientes que necessitaram de 3 procedimentos 44

ANEXO 5 - Acompanhamento tardio 45 
RESUMO 
Neves, C.C. Acidentes Cirúrgicos na Colecistectomia por Laparotomia. São Paulo, 2003. Dissertação (Mestrado) - Faculdade de Medicina da Universidade de São Paulo.

Estudos recentes estimam que a maioria das estenoses benignas é de natureza iatrogênica podendo ser evitada. A incidência de lesões graves dos ductos biliares durante a colecistectomia é de aproximadamente 1/300 a $1 / 500$ procedimentos $(0,2$ a $0,3 \%)$. Estas lesões podem resultar em coleperitôneo, fístula biliar, estenose de via biliar ou associação dessas complicações. O objetivo deste trabalho foi de relatar os casos de lesões de vias biliares tratados no Serviço de Cirurgia da Faculdade de Medicina da Universidade Federal de Goiás. Foram estudados retrospectivamente 31 pacientes, de 1990 a 2002, com diagnóstico de lesões de vias biliares pós colecistectomia. 29 pacientes $(93,5 \%)$ eram do sexo feminino e $2(6,5 \%)$ eram do sexo masculino. A idade variou de 21 a 80 anos com média de 46,6 anos. Pacientes provenientes da capital do Estado de Goiás eram em número de $12(38,7 \%)$ enquanto $19(61,2 \%)$ eram provenientes de outras regiões do país. A colecistectomia foi realizada eletivamente em 24 pacientes $(77,4 \%)$ enquanto em 7 pacientes $(22,5 \%)$ foi realizada cirurgia de urgência. A via de acesso através de incisão subcostal foi empregada em $70,9 \%$ dos pacientes e a via longitudinal (mediana e paramediana direita) em $29,1 \%$. As principais manifestações clínicas foram icterícia em $87,9 \%$ dos pacientes, fístula biliocutânea em $29 \%$, peritonite em $29,0 \%$, colangite em $29 \%$ e sepse em $6,4 \%$ dos pacientes. A Colangiografia Retrógrada Endoscópica foi o exame mais empregado para o diagnóstico da lesão de via biliar. As lesões dos ductos biliares foram classificadas segundo a classificação proposta por Strasberg. Aproximadamente metade $(54,8 \%)$ das lesões de vias biliares foram classificadas como Strasberg $E_{3}$ e $E_{4}$. A técnica cirúrgica mais utilizada para o tratamento das lesões de vias biliares 
foi a Hepaticojejunoanastomose, com alça jejunal exclusa em Y de Roux, em $20(64,5 \%)$ pacientes. Os 4 óbitos foram em lesões totais proximais. As lesões parciais evoluíram melhor a longo prazo. 
ABSTRACT 
Neves, C.C. Bile duct injuries during open cholecystectomy. São Paulo, 2003. Dissertação (Mestrado) - Faculdade de Medicina da Universidade de São Paulo.

Recent studies have estimated that most of the benign biliary stenosis are iatrogenic in nature, and can thus be prevent. The incidence of severe injury to the biliary ducts during a cholecystectomy is of approximately $1 / 300$ to $1 / 500$ procedures $(0.2-0.3 \%)$. These lesions can result in choleperitoneum, biliary fistula, stenosis of the biliary tract or an association of these complications. The objective of this paper was to report on biliary tract injuries treated at the Surgical Unit of the School of Medicine of the Federal University of Goias. Thirty-one patients with a diagnosis of biliary tract lesions, post cholecistectomy, were studied, retrospectively, from 1990 to 2002. Twenty-nine individuals (93.5\%) were female, and two (6.4\%), males. Their age ranged from 21 to 80 years (average of 46.6 years). Twelve $(38.7 \%)$ individuals came from the capital of State of Goias and $19(61.2 \%)$, from other regions. Cholecystectomy was performed as an elective procedure in $24(77.4 \%)$ patients, and $7(22.5 \%)$ underwent emergency surgery. A subcostal incision was used in $70.9 \%$ of the cases, while the longitudinal approach was used in $29.1 \%$. The main clinical manifestations were jaundice in $87.9 \%$, cutaneous fistula in $29 \%$, peritonitis in $29 \%$, cholangitis in $29 \%$ and sepsis in $6.4 \%$ of the patients. The Endoscopic Retrograde Cholangiography was the diagnostic test most frequently ordered. Biliary duct injuries were classified according to the criteria proposed by Strasberg. Aproximately half $(54.8 \%)$ of the injuries were classified as Strasberg $E_{3}$ and $E_{4}$. The most common surgical technique used in the treatment of this condition was a Roux-en-Y hepaticojejunostomy 
in twenty (64.5\%). Four patients with proximal injuries died. Long term followup disclosed better results with partial injuries. 


\section{INTRODUÇÃO}




\subsection{Introdução}

A prevalência da litíase biliar varia nos diversos países, nas diferentes raças e nos métodos de estudo. As estatísticas de autópsias sempre apresentaram resultados elevados, com uma incidência em torno de $15 \%$ a 40\%, passando para 50\% em mulheres idosas de vários países (HEATON, 1973; BOUCHIER, 1988; SAMPLINER et al., 1970; TORVIK et al., 1960).

As pesquisas populacionais radiográficas objetivando precisar casos de litíase vesicular são raras e seus resultados são sujeitos a críticas. Dados levantados na América do Norte concluem que a prevalência ocorre em torno de $11 \%$ entre os homens e $16,7 \%$ entre as mulheres com idade de 35 a 60 anos (CHAPMAN, 1996 e FRIEDMAN et al., 1966). No Reino Unido esta prevalência oscila entre $6,2 \%$ e $12,1 \%$ nos pacientes com idade entre 45 a 69 anos (HEATON, 1973).

As pesquisas de casos clínicos apresentam resultados menos consistentes. Metman et al (1981), em estudo realizado na França em 1976, encontrou uma prevalência de 6,2\% e uma incidência anual de 1,1\%.

Considerando que, na França, encontrou 4 milhões de litiásicos e 200.000 novos casos a cada ano, Levy (1988), afirmou que 1 mulher entre 3 , e 1 homem entre 5, é ou será litiásico um dia.

As pesquisas que se utilizam do método ultra-sonográfico dão resultados mais aproximados das prevalências. Num desses estudos, envolvendo 1.930 indivíduos com idade entre 18 e 65 anos, em Sirmione, 
pequena cidade da Itália, Barbara et al (1987), está provada litíase vesicular em torno de $6,7 \%$ dos homens e $14,6 \%$ entre as mulheres. A prevalência de litíase aumentava, com a idade, em ambos os sexos.

Contrastando com tais evidências encontramos, nos países da África, uma taxa menor que 5\%. Na comunidade Massai, no Leste do continente africano, a taxa de prevalência é quase zero (BISS et al., 1971).

A prevalência de colelitíase, no Brasil, foi avaliada na cidade de Curitiba-PR, com uma amostragem de 1.000 indivíduos num universo de 2.500.000 de habitantes. Os estudados, após entrevista médica, foram imediatamente submetidos a exame ultra-sonográfico. À avaliação dos resultados, $905(90,5 \%)$ apresentaram vesícula normal; 2 (0,2\%) tinham pólipos de vesícula, e $93(6,3 \%)$ tinham colelitíase. A prevalência de colelitíase foi aumentando de 2,4\% nos indivíduos com idade entre 20 a 29 anos, para $27,5 \%$ entre os indivíduos com idade acima de 70 anos. Esta predominância aumentou entre as mulheres nulíparas grávidas de 4\% para $34,6 \%$ em multíparas. A prevalência por sexo foi de $12,9 \%$ entre as mulheres; e de $5,4 \%$ entre os homens.

O método ultra-sonográfico aqui empregado, apresentou uma precisão de $98 \%$ para o diagnóstico de colelitíase. Tal precisão leva à conclusão de ser o ultra-sonográfico o melhor método para determinar a prevalência de colelitíase em uma população (FRIEDMAN et al., 1966; ATTILI et al., 1995). É possível, portanto, afirmar que a ocorrência de colelitíase, no nosso país, é bastante elevada (COELHO, 1999). 
Sendo a colecistectomia o tratamento mais adequado para a maioria destes casos, esta é uma das operações mais freqüentemente realizadas em cirurgia do aparelho digestivo.

A colecistectomia foi realizada primeiramente por Langenbuch, em 1882. O seu emprego rotineiro no tratamento da colelitíase foi gradual e, a lesão de estruturas do pedículo biliar somente foi relatada por Mayo (1905), quando este descreveu dois casos de coledocoduodenostomia para tratar lesão de ducto colédoco ocorrida durante a realização de colecistectomia de rotina.

Nas complicações intra-operatórias, a abertura da vesícula biliar é um incidente freqüente, quer nas cirurgias por laparotomia, quer nas cirurgias por via laparoscópica; principalmente quando se trata de vesícula biliar com paredes comprometidas por processo inflamatório, ou quando se apresenta com grau diferente de necrose. Estes acidentes constituem um problema contornável.

O diagnóstico das estenoses biliares, no inicio do século $X X$, como conseqüência de lesão iatrogênica durante as colecistectomias, não era amplamente aceito. Tal postura retardava o diagnóstico que geralmente dependia da evolução natural do quadro clínico. O paciente evoluía com fístula biliar, colangite, icterícia ou outras condições como cirrose biliar secundária e, a partir daí, aventava-se a hipótese diagnóstica de lesão iatrogênica das vias biliares. Desta forma, a condição clínica do paciente com cirrose biliar e hipertensão portal - com todas as complicações dessas 
situações -, somada à pouca familiarização dos cirurgiões com tais lesões, deixava a desejar quanto ao grau de satisfação do tratamento.

Estudos recentes estimam que a maioria das estenoses benignas é de natureza iatrogênica podendo, portanto, ser evitadas (COLLINS, 1984, SCHIRMER, 1991). Estudo multicêntrico de Bismuth (1981), relata a incidência de 0,15 e $0,2 \%$ de lesões iatrogênicas da via biliar em colecistectomia por laparotomia. Diversos estudos apresentam índices elevados de lesões iatrogênicas da via biliar que deixaram de ser reconhecidas por ocasião das operações. Destes estudos destacam-se: Lahey et al (1950) refere um índice de até 90\%; Longmire (1966) estima este índice em torno de 37\%; Bismuth (1983) refere um índice de 45\%; Rosenquist et al (1996) avaliaram este índice em 53\%; e Penteado et al (2002) estimaram estes dados em $85 \%$. Portanto, a reoperação para tratamento de estreitamentos das lesões biliares constitui uma necessidade, na maioria dos casos.

A incidência das lesões dos ductos biliares caiu para aproximadamente 0 a $0,2 \%$, à medida que os cirurgiões tornaram-se mais experientes com a cirurgia aberta (MOOSA et al., 1992).

No final dos anos 80, Mühe (1986), na Alemanha; Dubois et al (1990); Perissat et al (1992), e Cushieri et al (1991), na França, introduziram a colecistectomia laparoscópica na Europa. Logo a seguir, foram acompanhados por Reddick et al (1991) e Olsen (1991), nos Estados Unidos. A incidência de lesões dos ductos biliares, nas colecistectomias por via laparoscópica, variava, inicialmente, de 0,5\% a 0,8\% (DEZIEL, 1993). 
A introdução desta técnica reacendeu a discussão sobre a possibilidade de lesão da via biliar. E nos motivou a rever os pacientes portadores de lesão iatrogênica de via biliar, tratados no Hospital das Clínicas da Faculdade de Medicina da Universidade Federal de Goiás. 


\section{OBJETIVO}


2.0 Objetivo

O objetivo do presente estudo é avaliar alterações e complicações decorrentes de lesões iatrogênicas dos ductos biliares após colecistectomia por laparotomia. Busca-se:

1. Estabelecer a freqüência de lesão das vias biliares (LVB).

2. Analisar os resultados imediatos e tardios da correção cirúrgica de lesões de vias biliares (LVB). 


\section{CASUÍSTICA E MÉTODOS}




\subsection{Casuística e Métodos}

\subsection{Casuística}

Foram estudados, retrospectivamente, os pacientes tratados por lesão iatrogênica da via biliar no período de janeiro de 1990 a dezembro de 2002. A investigação deu-se sobre o número de colecistectomias realizadas no Hospital das Clínicas da Universidade Federal de Goiás, neste intervalo.

Foram tratados 17 (55\%) pacientes provenientes da capital do estado de Goiás, e 14 (45\%) provenientes de outras regiões do pais, constituindo um total de 31 pacientes. Destes, $29(93,5 \%)$ do sexo feminino e $2(6,5 \%)$ do sexo masculino. A idade média dos pacientes foi de 46,6. Observou-se desvio padrão (DP) de 11,59 anos com variação de 21 a 80 anos de idade. A maioria dos pacientes (55\%) era procedente da capital do Estado de Goiás, e $45 \%$ de outras regiões do país.

\subsubsection{Critérios de inclusão}

Os pacientes submetidos a colecistectomia foram incluídos obedecendo os seguintes critérios:

- Pacientes submetidos a colecistectomia, procedentes de Goiás e de outros Estados 
- Idade entre 21 e 80 anos

- Pacientes submetidos a colecistectomia no HC da UFG com exames complementares e descrição de cirurgia evidenciando lesão de vias biliares

- Pacientes com lesão de via biliar operados em outros serviços e encaminhados para tratamento no $\mathrm{HC}$ da UFG 


\subsection{Método}

Estudo retrospectivo analisando dados referentes a:

- Número de colecistectomias realizadas no período de 1990 a 2002

- Idade e sexo

- Tipo de incisão

- Quadro clínico

- Exames complementares

- Altura da lesão

- Tratamento

- Resultados imediatos

- Evolução tardia

- Análise estatística 
RESULTADOS 


\subsection{Resultados}

4.1 Resultados referentes ao número de colecistectomias realizadas no período estudado.

Tabela 1 - Número de pacientes operados de 1990 a 2002

\begin{tabular}{lcrrrr}
\hline \multirow{2}{*}{ Sexo } & \multicolumn{4}{c}{$\mathbf{n}$} & \multirow{2}{*}{ TOTAL } \\
\cline { 2 - 5 } Feminino & 961 & $82,4 \%$ & 165 & $84,7 \%$ & $\mathbf{1 . 1 2 6}$ \\
Masculino & 205 & $17,5 \%$ & 30 & $15,3 \%$ & 235 \\
\hline TOTAL & $\mathbf{1 . 1 6 6 ( 8 5 , 7 \% )}$ & $\mathbf{1 9 5}(\mathbf{1 4 , 3} \%)$ & $\mathbf{1 . 3 6 1}$ \\
\hline
\end{tabular}

$\mathrm{n}=$ Número de pacientes $\quad \mathrm{p}=0,063$

O estudo estatístico não revelou diferença significativa entre a ocorrência de cirurgia de urgência em relação aos sexos.

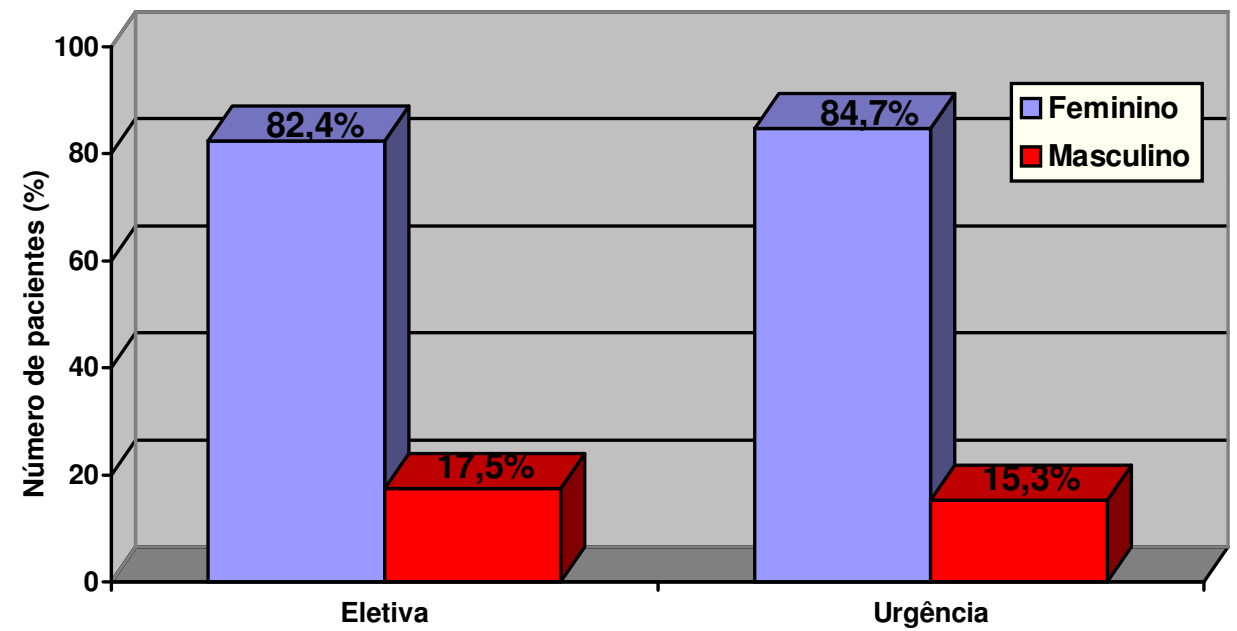

Figura 1 - Número de pacientes operados de 1990 a 2002 
Do total de 1.361 pacientes operados por colelitíase, 12 (0,82\%) apresentavam LVB.

Tabela 2 - Incidência de lesão relacionada à indicação da colecistectomia

\begin{tabular}{lcr}
\hline Indicação da colecistectomia & LVB - HC $(\%)$ & Total \\
\hline Eletiva & $9(75)$ & 1.166 \\
Urgência & $3(25)$ & 195 \\
\hline Total & $12(0,9)$ & 1.361 \\
\hline$p=0,161$ & &
\end{tabular}

A análise estatística não revelou diferença significativa entre as ocorrências de lesão em operações eletivas e de urgência

Tabela 3 - Incidência de lesão quanto aos sexos

\begin{tabular}{lcc}
\hline Sexo & LVB $(\%)$ & Total \\
\hline Masculino & $1(8,3)$ & 235 \\
Feminino & $11(91,7)$ & 1126 \\
\hline Total & 12 & 1361 \\
\hline $\mathrm{p}=0,257$ & &
\end{tabular}

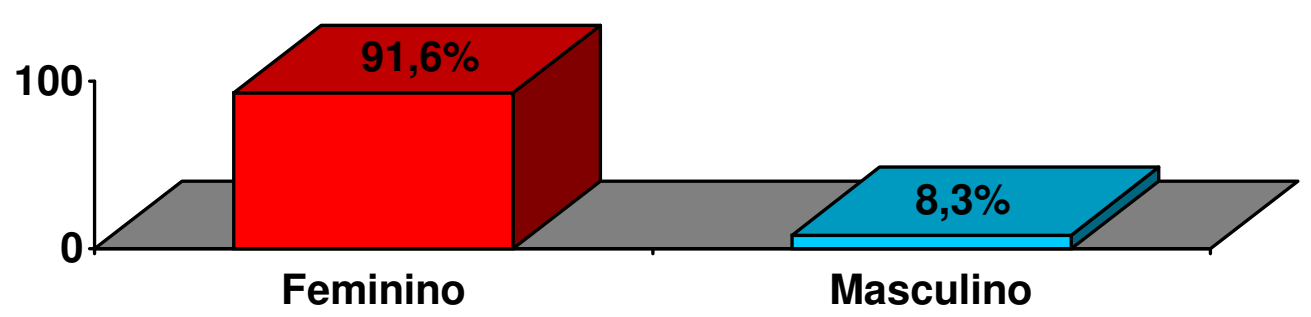

Figura 2 - Distribuição dos pacientes quanto ao sexo

A análise estatística não revelou diferença significativa entre as ocorrências de lesão nos sexo masculino e feminino. 
Tabela 4 - Incidência de lesão relacionada à colecistectomia realizada em outras regiões da Federação

\begin{tabular}{lc}
\hline Indicação da colecistectomia & LVB $(\%)$ \\
\hline Eletiva & $15(78,9)$ \\
Urgência & $4(21,1)$ \\
\hline Total & $19(100,0)$ \\
\hline
\end{tabular}

Tabela 5 - Incidência de lesão quanto aos sexos - pacientes procedentes de outros Estados

\begin{tabular}{lc}
\hline Sexo & LVB $(\%)$ \\
\hline Feminino & $18(94,7)$ \\
Masculino & $1(5,3)$ \\
\hline Total & $19(100,0)$ \\
\hline
\end{tabular}

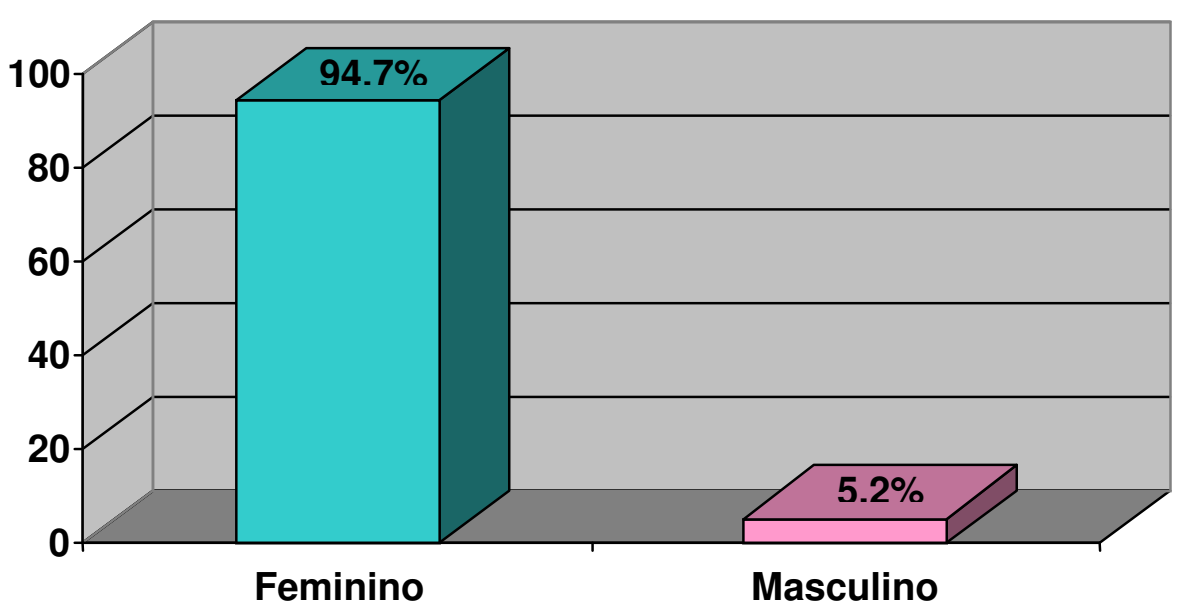

Figura 3 - Distribuição dos pacientes de outras regiões quanto ao sexo 
Tabela 6 - Distribuição dos pacientes por faixa etária.

\begin{tabular}{lc}
\hline Faixa etária & Número pacientes \\
\hline $21-30$ & 3 \\
$31-40$ & 7 \\
$41-50$ & 10 \\
$51-60$ & $6^{\star}$ \\
$61-70$ & $4^{*}$ \\
$71-80$ & 1 \\
\hline Total & $\mathbf{3 1}$ \\
\hline
\end{tabular}

* Paciente do sexo masculino

Média $=46,6$ anos

$\mathrm{DP}=11,59$ anos

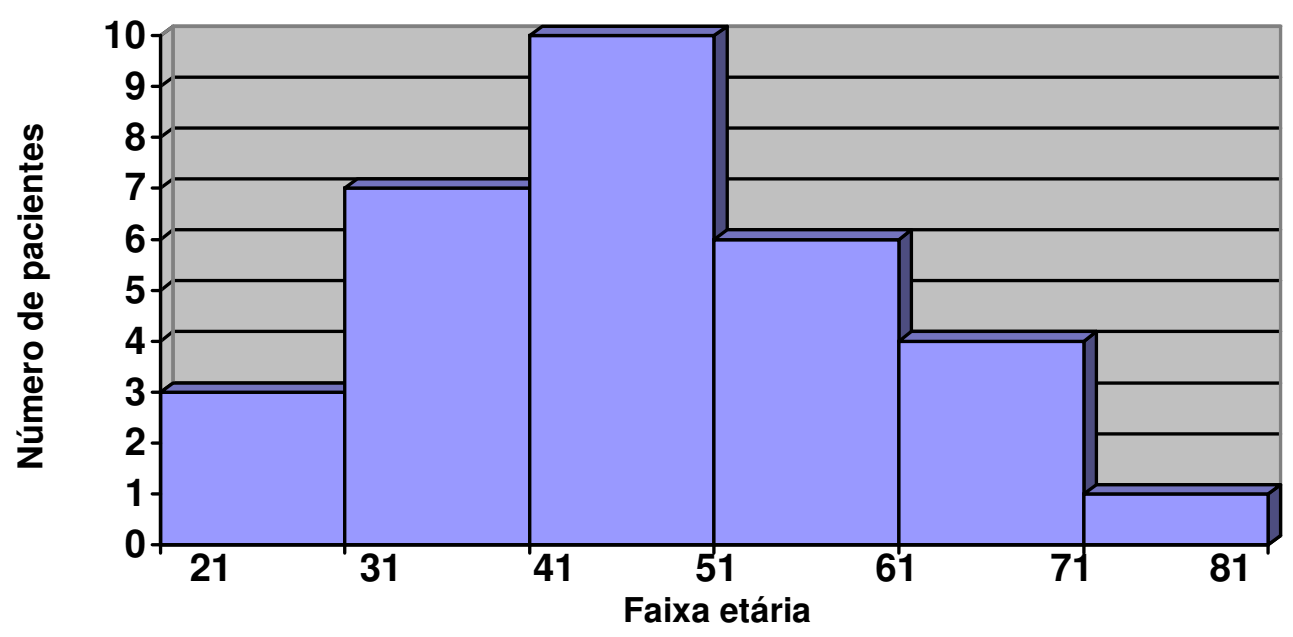

Figura 4 - Distribuição dos pacientes por faixa etária. 
A incisão mais utilizada foi a subcostal tanto no grupo de urgência quanto no grupo eletivo (Tabela 7).

Tabela 7 - Tipo de incisão utilizada na colecistectomia e caráter da cirurgia.

\begin{tabular}{lccc}
\hline \multirow{2}{*}{ Caráter } & \multicolumn{2}{c}{ Incisão } & \multirow{2}{*}{ Total } \\
\cline { 2 - 3 } & Longitudinal & Subcostal & \\
\hline Eletiva & 8 & 18 & 26 \\
Urgência & 1 & 4 & 5 \\
\hline TOTAL & $\mathbf{9}$ & $\mathbf{2 2}$ & $\mathbf{3 1}$ \\
\hline
\end{tabular}

$p=0,387$

A análise estatística não revelou diferença significativa entre o tipo de incisão e a indicação eletiva/urgência.

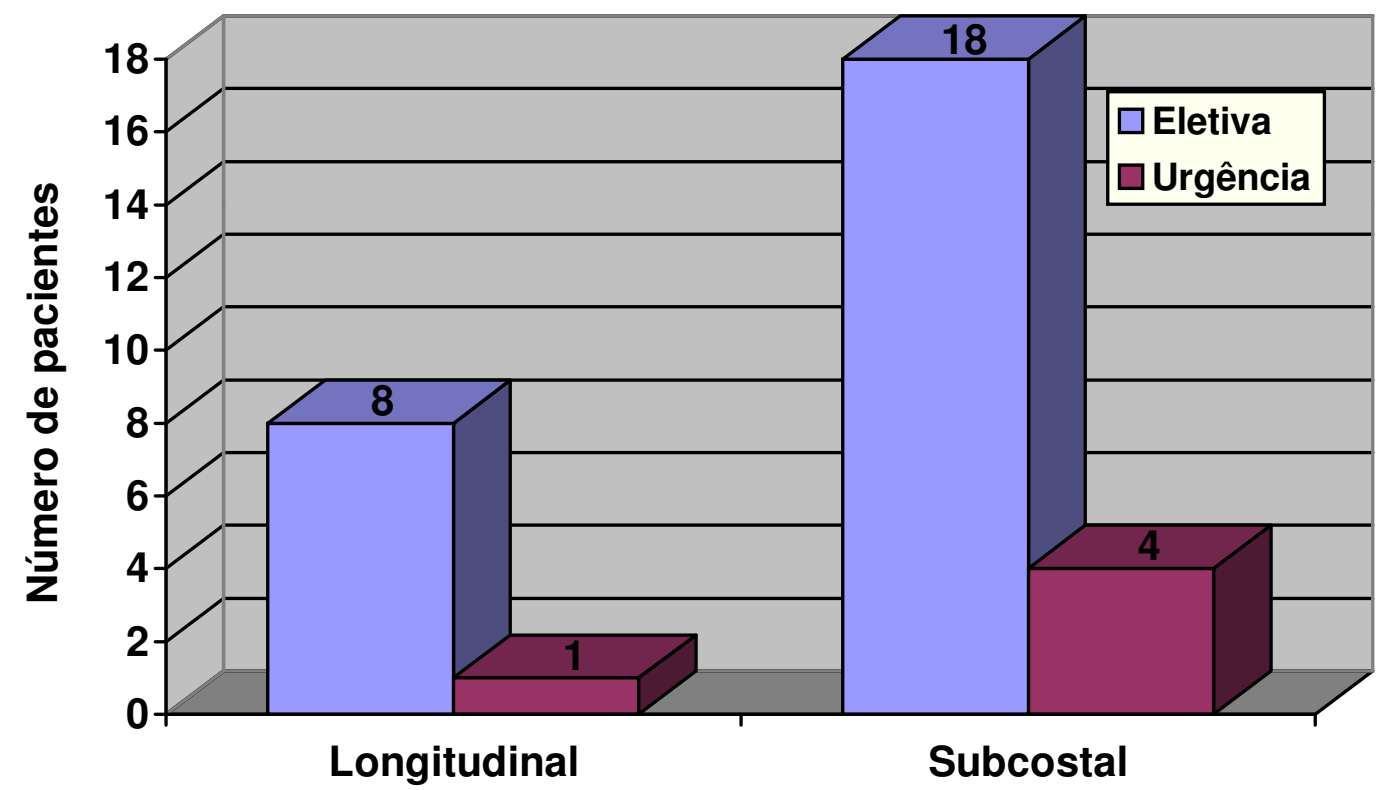

Figura 5 - Tipo de incisão utilizada na colecistectomia e caráter da cirurgia. 
Tabela 8 - Sinais e sintomas dos pacientes pós-colecistectomia.

\begin{tabular}{lcc}
\hline Sinais e sintomas & $\mathbf{n}$ & $(\%)$ \\
\hline Icterícia & 27 & 87,1 \\
Peritonite & 9 & 29,0 \\
Fístula cutânea & 9 & 29,0 \\
Colangite & 9 & 29,0 \\
Sepse & 2 & 6,5 \\
\hline
\end{tabular}

$\mathrm{n}=$ Número de pacientes

Alguns pacientes apresentaram mais de uma manifestação.

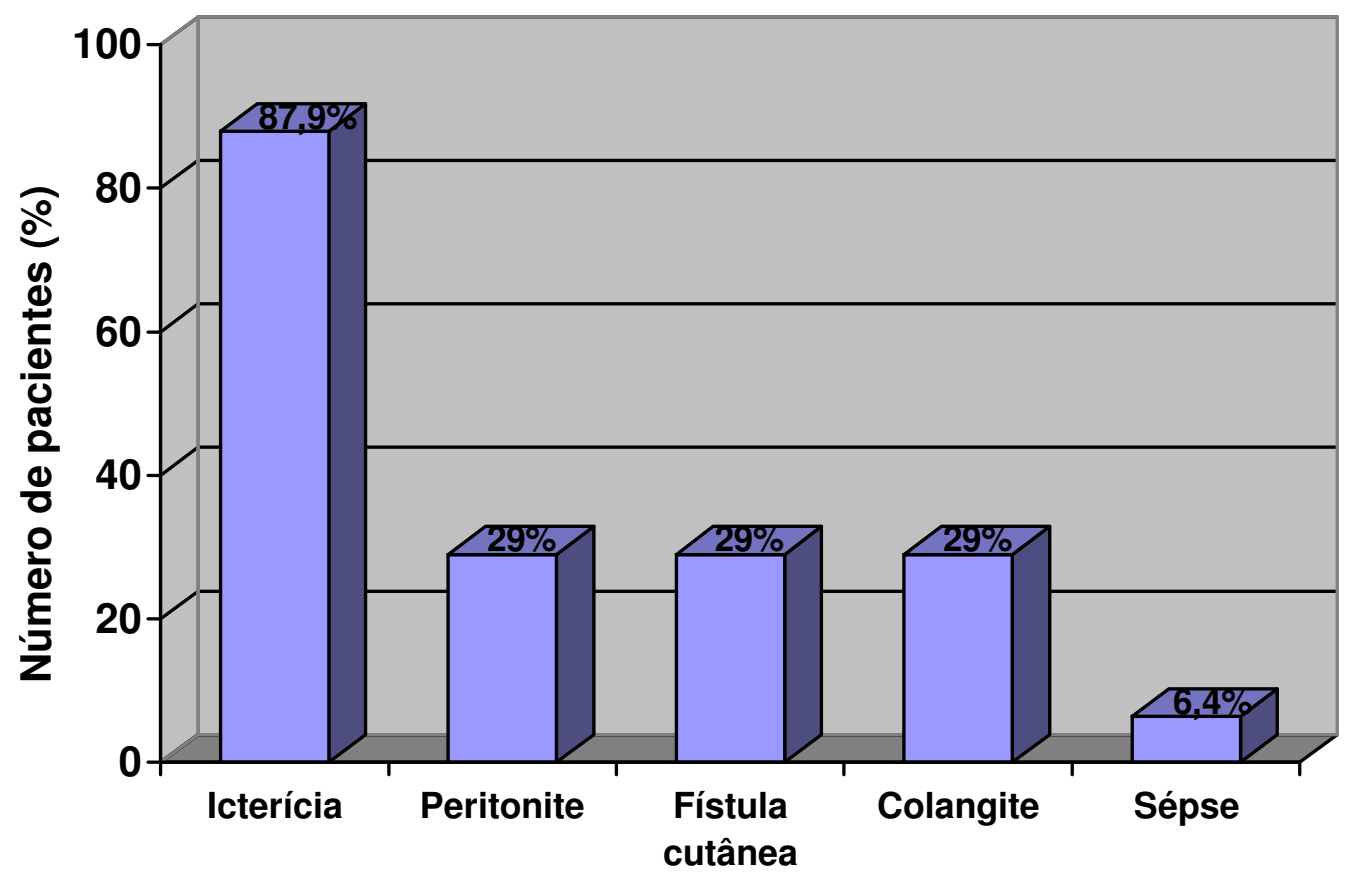

Figura 6 - Sinais e sintomas dos pacientes colecistectomizados

A icterícia e a peritonite foram os sinais e sintomas mais freqüentes nesta série de pacientes (Tabela 8). 
Tabela 9 - Pacientes submetidos a colangiografia

\begin{tabular}{lcc}
\hline Colangiografia & $\mathbf{n}$ & $\%$ \\
\hline Colangiografia intra-operatória & 4 & 12,9 \\
Colangiografia endoscópica retrógrada & 8 & 25,8 \\
Colangiografia transparietohepática & 2 & 6,5 \\
Colangio-ressonância magnética & 1 & 3,2 \\
Colangiografia pré-operatória & 1 & 3,2 \\
\hline TOTAL & $\mathbf{1 6}$ & $\mathbf{5 1 , 6}$ \\
\hline
\end{tabular}

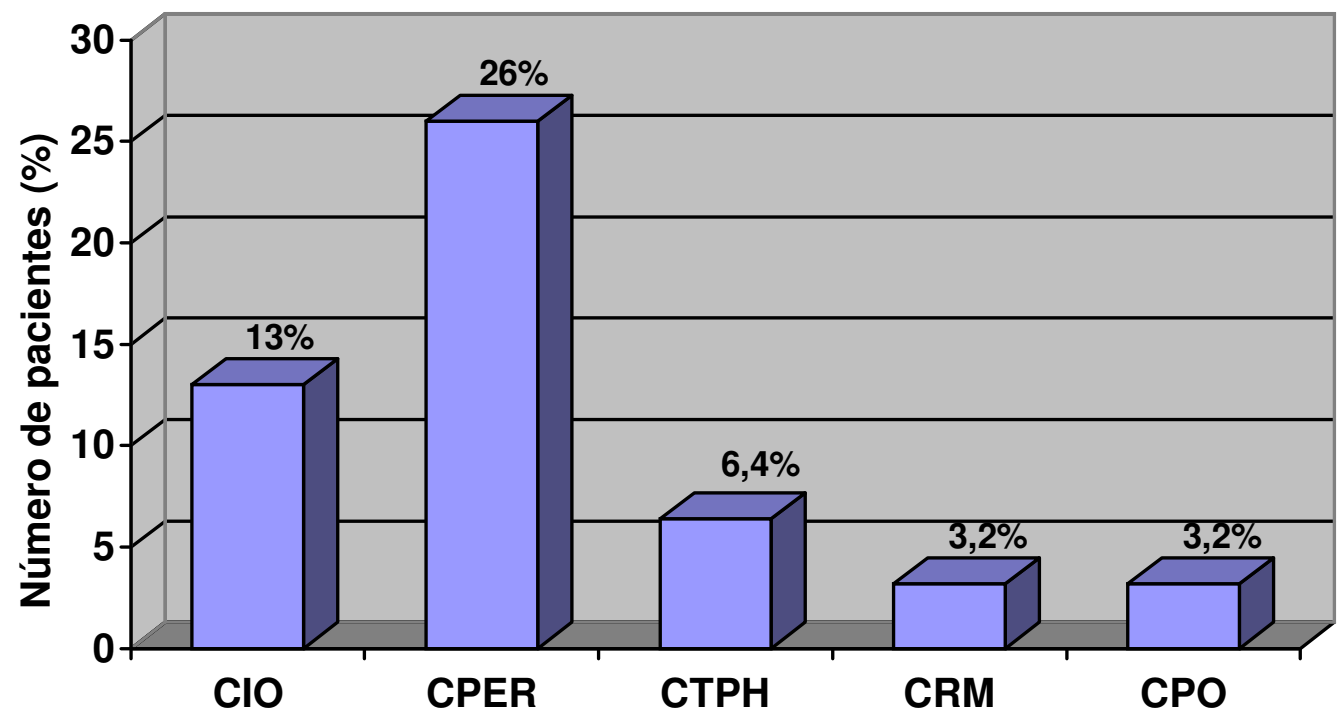

Figura 7 - Pacientes submetidos a colangiografia

As lesões foram classificadas de acordo com o método proposto por Strasberg et al., em 1996 (Tabela 10). 
Tabela 10 - Classificação das lesões segundo Strasberg

\begin{tabular}{lcc}
\hline Tipo de lesão & $\mathbf{n}$ & $\%$ \\
\hline $\mathrm{SE}_{1}$ & 3 & 9,7 \\
$\mathrm{SE}_{2}$ & 3 & 9,7 \\
$\mathrm{SE}_{3}$ & 8 & 25,8 \\
$\mathrm{SE}_{4}$ & 9 & 29,0 \\
$\mathrm{SB}$ & 3 & 9,7 \\
$\mathrm{SD}$ & 5 & 16,1 \\
\hline Total & $\mathbf{3 1}$ & $\mathbf{1 0 0 , 0}$ \\
\hline
\end{tabular}

$\mathrm{n}=$ Número de pacientes
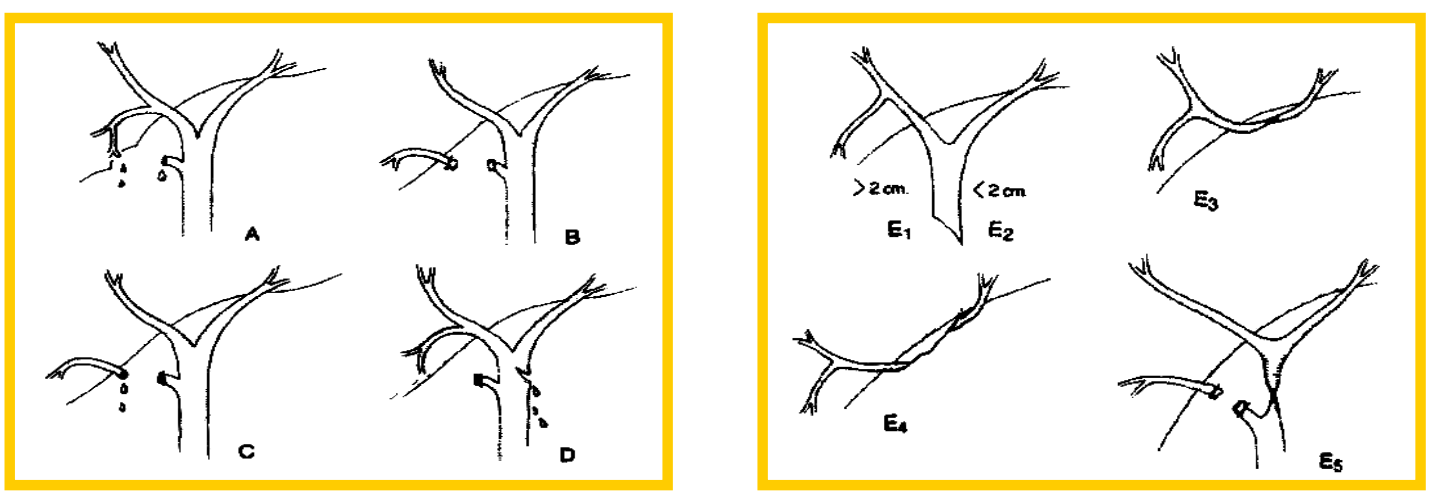

Figura 8 - Classificação das LVB segundo Strasberg.

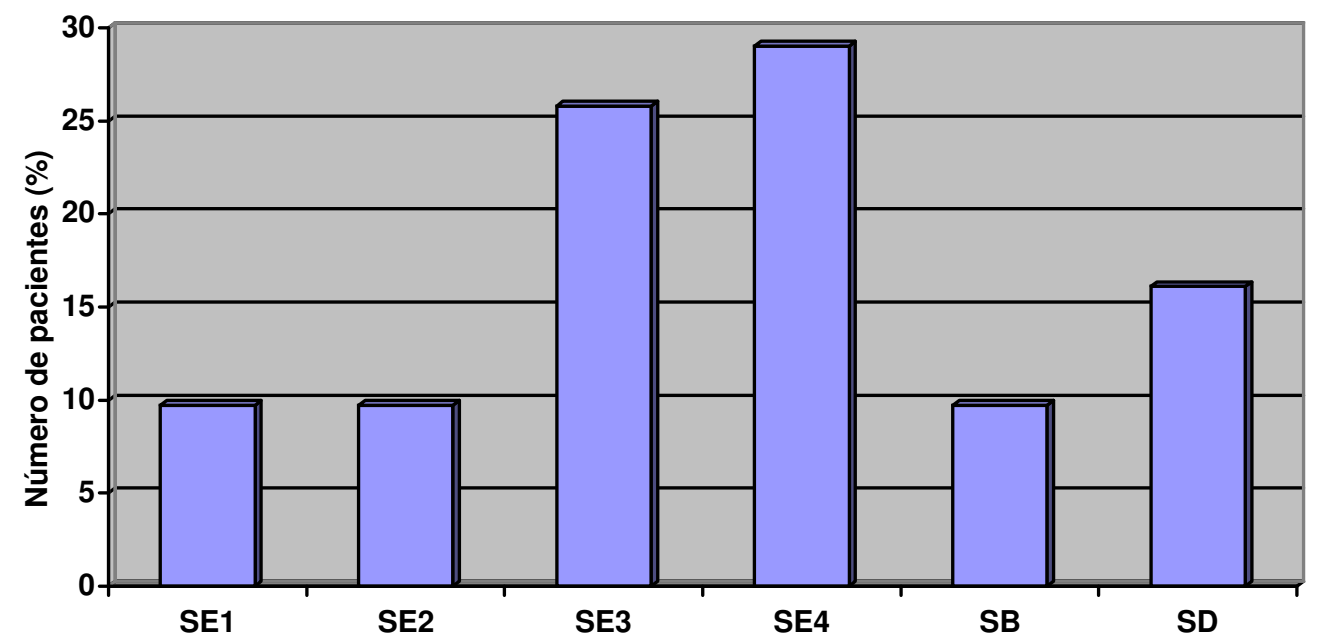

Figura 9 - Classificação das lesões segundo Strasberg 
Tabela 11 - Ocasião do diagnóstico e tratamento da lesão da via biliar por colecistectomia

\begin{tabular}{lcc}
\hline Tempo do diagnóstico & $\mathbf{n}$ & $\%$ \\
\hline Durante a operação & 1 & 3,2 \\
Até 30 dias & 13 & 41,9 \\
Após 30 dias & 17 & 54,9 \\
\hline Total & 31 & 100,0 \\
\hline
\end{tabular}

$\mathrm{n}=$ Número de pacientes

Tabela 12 - Tratamento cirúrgico das lesões das vias biliares.

\begin{tabular}{lcc}
\hline Tipo de correção & $\mathbf{n}$ & $\%$ \\
\hline Hepaticojejunoanastomose & 20 & 64,5 \\
Coledocoduodenoanastomose & 2 & 6,5 \\
Drenagem da VB & 9 & 29,0 \\
\hline Total & $\mathbf{3 1}$ & $\mathbf{1 0 0 , 0}$ \\
\hline
\end{tabular}

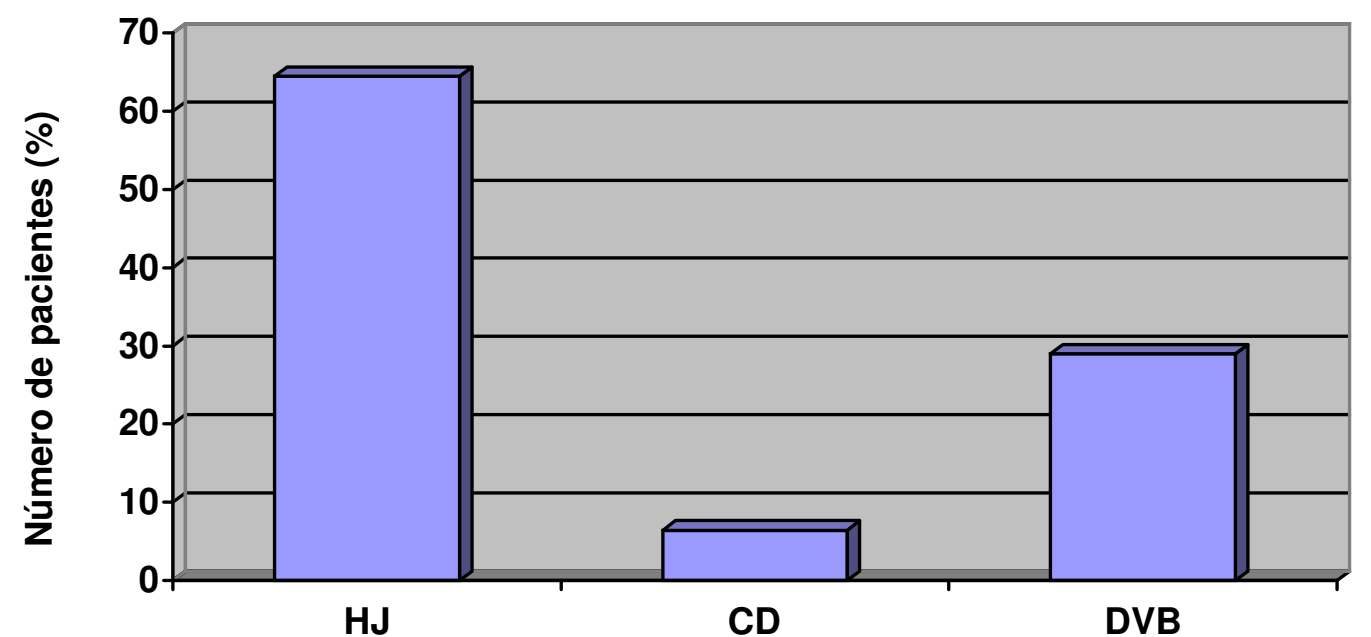

Figura 10 - Tratamento cirúrgico das lesões das vias biliares e o tipo de lesão

A hepaticojejunoanastomose foi o procedimento utilizado em 20 pacientes; a drenagem biliar foi utilizada em 9 pacientes e, em 2 pacientes foi utilizada a coledocoduodenoanastomose (Tabela 12). 
Tabela 13 - Tratamento por meio de drenagem da via biliar.

\begin{tabular}{lcc}
\hline Classificação de Strasberg & $\mathbf{n}$ & $\%$ \\
\hline $\mathrm{SD}$ & 5 & 16,1 \\
$\mathrm{SB}$ & 2 & 6,5 \\
$\mathrm{SE}_{4}$ & 2 & 6,5 \\
\hline Total & $\mathbf{9}$ & $\mathbf{2 9 , 0 3}$ \\
\hline
\end{tabular}

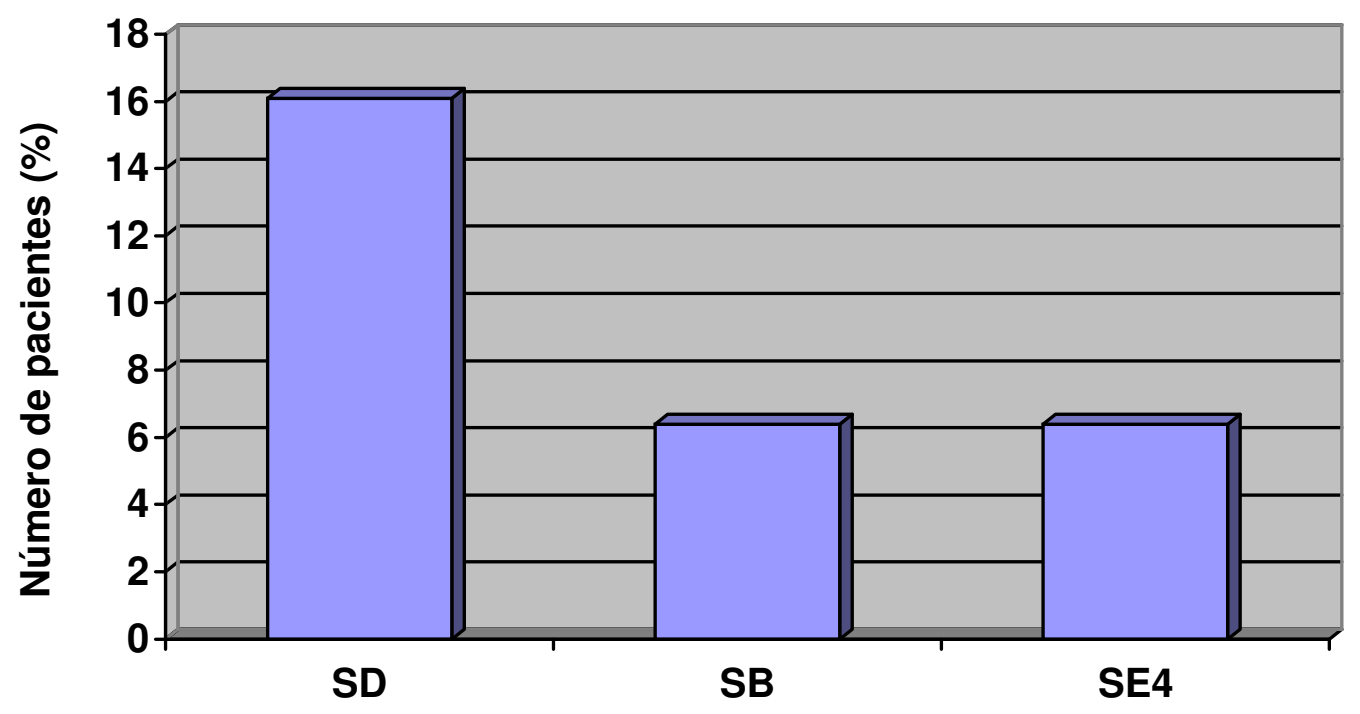

Figura 11 - Drenagem da via biliar

Tabela 14- Tempo de permanência de drenos

\begin{tabular}{lccccc}
\hline Drenos & Tratamento & Moldagem & $\begin{array}{c}\text { Tempo de } \\
\text { permanência (dias) }\end{array}$ & $\mathbf{n}$ & $\%$ \\
\hline Kehr & 7 & 4 & $20-90$ & 11 & 35,5 \\
Nelaton & - & 5 & $7-45$ & 5 & 16,1 \\
${ }^{*}$ Foley & 2 & - & $2-40$ & 2 & 6,5 \\
\hline Total & $\mathbf{9}$ & $\mathbf{9}$ & & $\mathbf{1 8}$ & $\mathbf{5 8 , 1}$ \\
\hline
\end{tabular}

* Óbitos 
Tabela 15 - Complicações pós-operatórias imediatas. Óbitos

\begin{tabular}{|c|c|c|c|c|c|c|c|c|}
\hline \multirow{2}{*}{ Tipo de complicação } & \multicolumn{6}{|c|}{ Número de pacientes por tipo de lesão } & \multirow{2}{*}{ Total } & \multirow{2}{*}{$\%$} \\
\hline & $\mathrm{SE}_{1}$ & $\mathrm{SE}_{2}$ & $\mathrm{SE}_{3}$ & $\mathrm{SE}_{4}$ & SB & SD & & \\
\hline Óbito por sepse & - & - & 1 & 3 & - & - & 4 & 12,9 \\
\hline Infecção de ferida & 2 & 2 & 1 & 1 & 2 & 1 & 9 & 29,0 \\
\hline Fístula biliar & - & - & 2 & 2 & - & 1 & 5 & 16,1 \\
\hline $\begin{array}{l}\text { Abscesso intra- } \\
\text { cavitário }\end{array}$ & - & - & 2 & - & - & - & 2 & 6,4 \\
\hline Ascite & - & - & - & 1 & - & - & 1 & 3,2 \\
\hline Diarréia & 1 & - & 1 & 1 & - & - & 3 & 9,7 \\
\hline Total & 3 & 2 & 7 & 8 & 2 & 2 & 24 & 77,4 \\
\hline
\end{tabular}

Embora sem significado estatístico, alguns pacientes apresentaram mais de uma complicação.

Tabela 16 - Mortalidade em relação à ocasião do tratamento

\begin{tabular}{lccccc}
\hline Tratamento & Sobrevida & $\%$ & Óbito & $\%$ & Total \\
\hline Em 30 dias & 13 & 41,0 & 1 & 3,2 & 14 \\
Após 30 dias & 14 & 45,1 & 3 & 9,6 & 17 \\
\hline \multicolumn{1}{c}{ Total } & $\mathbf{2 7}$ & $\mathbf{8 7 , 0}$ & $\mathbf{4}$ & $\mathbf{1 2 , 9}$ & $\mathbf{3 1}$ \\
\hline $\begin{array}{l}\mathrm{p}=0,303 \\
\text { Óbitos durante a internação ocorreram nos casos de lesão mais alta, } \mathrm{SE}_{3} \mathrm{e} \\
\mathrm{SE}_{4 .}\end{array}$
\end{tabular}


Tabela 17 - Doenças associadas à admissão para tratamento cirúrgico das LVB

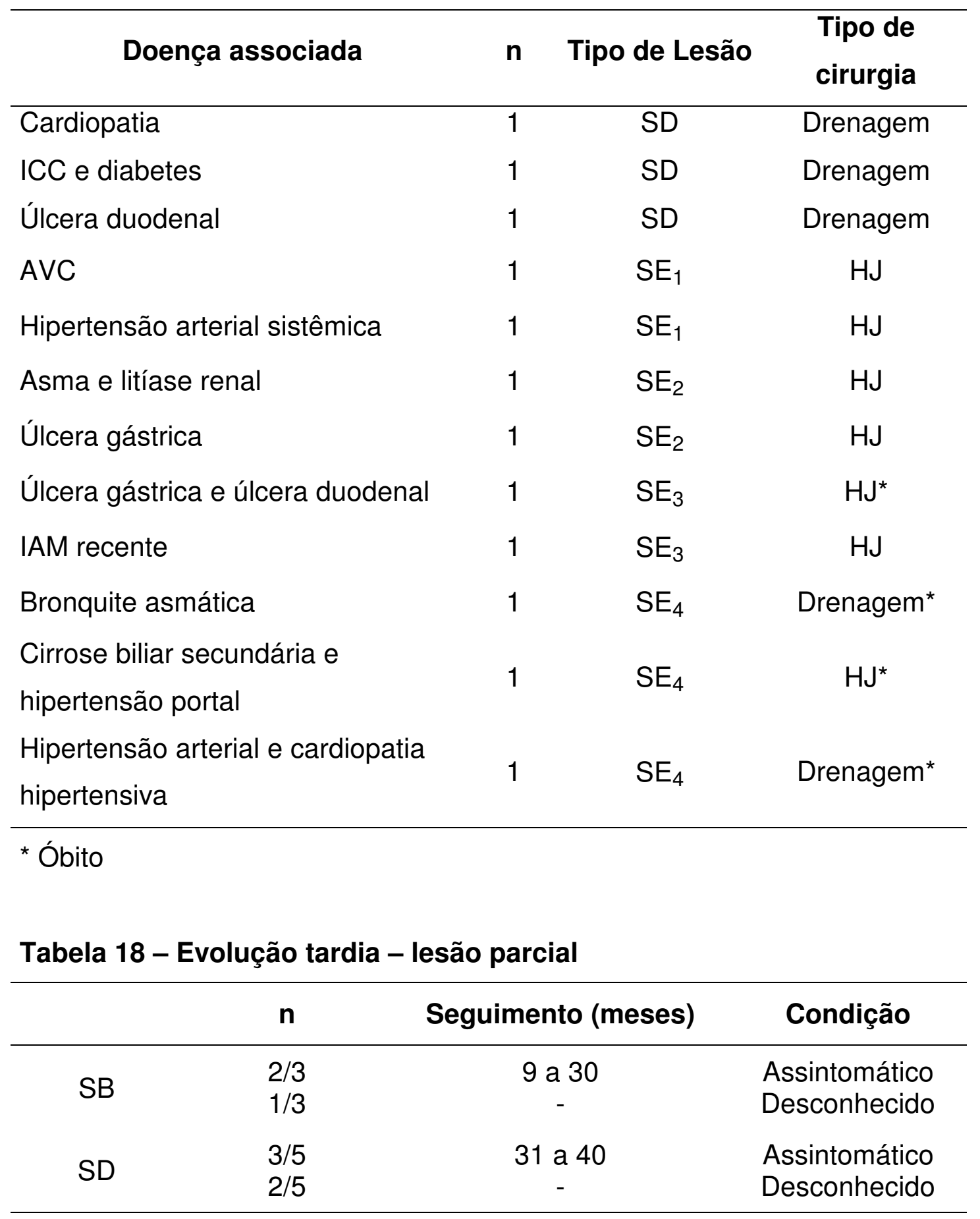


Tabela 19 - Evolução tardia - lesão total

\begin{tabular}{cccc}
\hline & $\mathbf{n}$ & Seguimento (meses) & Condição \\
\hline $\mathrm{SE}_{1}$ & $3 / 3$ & 32 a 60 & Assintomáticos \\
\hline \multirow{2}{*}{$\mathrm{SE}_{2}$} & $1 / 3$ & 41 & Enzimas alteradas \\
& $2 / 3$ & - & Desconhecidos \\
\hline \multirow{3}{*}{$\mathrm{SE}_{3}$} & $4 / 7$ & 19 a 68 & Assintomáticos \\
& $1 / 7$ & 5 & Enzimas alteradas \\
& $1 / 7$ & - & Desconhecido \\
& $1 / 7$ & - & Óbito \\
$\mathrm{SE}_{4}$ & $3 / 7$ & 2 a 67 & Assintomáticos \\
& $1 / 7$ & 120 & RV \\
& $2 / 7$ & - & Desconhecidos \\
& $1 / 7$ & - & Óbito \\
\hline
\end{tabular}

A análise estatística não revelou diferença significativa entre a mortalidade dos pacientes tratados nos primeiros 30 dias (complicações imediatas) e dos pacientes tratados após 30 dias.

A hipertensão arterial e a doença ulcerosa péptica foram as doenças mais freqüentemente encontradas em 5 (16,1\%) pacientes (Tabela 15). Dois pacientes morreram em decorrência do tratamento cirúrgico em pósoperatório imediato; e outros dois morreram em razão do tratamento cirúrgico relacionado a doenças associadas.

Os pacientes foram submetidos a cirurgias de drenagem ou derivação para alça intestinal exclusa (Tabelas 12 e 13). Não foi possível acompanhar, efetivamente, 9 dos 27 pacientes que receberam alta hospitalar. $O$ seguimento tardio incluiu 18 pacientes; 11 foram avaliados em consulta e exames ambulatoriais. Informações por telefone foram obtidas de outros 7 pacientes; uma faleceu após 12 anos; outro, que já apresentava cirrose na ocasião do tratamento, não aceita novos exames (RV), bem como outros 5 , que estão em outras localidades distantes. 
Tabela 20 - Complicações imediatas

\begin{tabular}{lccc}
\hline $\begin{array}{c}\text { Tipo de } \\
\text { lesão }\end{array}$ & $\mathbf{n}$ & Período (dias) & Acompanhamento \\
\hline $\mathrm{SE}_{3}$ & 1 & 7 & Óbito \\
$\mathrm{SE}_{4}$ & 1 & 40 & Óbito \\
$\mathrm{SE}_{4}$ & 1 & 1 & Óbito \\
\hline Total & $\mathbf{3}$ & - & $\mathbf{3}$ \\
\hline
\end{tabular}

Tabela 21 - Acompanhamento tardio

\begin{tabular}{|c|c|c|c|c|}
\hline $\begin{array}{c}\text { Tipo de } \\
\text { lesão }\end{array}$ & $\mathrm{n}$ & $\begin{array}{c}\text { Evolução } \\
\text { (meses) }\end{array}$ & Condição & $\begin{array}{c}\text { Exames } \\
\text { complementares }\end{array}$ \\
\hline SB & $\begin{array}{l}2 \\
1\end{array}$ & $\begin{array}{c}9-30 \\
\text { Desconhecido }\end{array}$ & Assintomático & RV \\
\hline SD & $\begin{array}{l}1 \\
1 \\
1 \\
1 \\
1 \\
\end{array}$ & $\begin{array}{c}31 \\
\text { Desconhecido } \\
37 \\
40 \\
\text { Desconhecido }\end{array}$ & $\begin{array}{c}\text { Assintomático } \\
- \\
\text { Assintomático } \\
\text { Assintomático } \\
\text { - }\end{array}$ & $\begin{array}{c}\text { Enzimas normais } \\
- \\
- \\
\mathrm{RV} \\
- \\
\end{array}$ \\
\hline $\mathrm{SE}_{1}$ & $\begin{array}{l}1 \\
1 \\
1\end{array}$ & $\begin{array}{l}48 \\
60 \\
32\end{array}$ & $\begin{array}{l}\text { Assintomático } \\
\text { Assintomático } \\
\text { Assintomático }\end{array}$ & $\begin{array}{c}\text { Enzimas normais } \\
\text { Enzimas normais } \\
\text { RV }\end{array}$ \\
\hline $\mathrm{SE}_{2}$ & $\begin{array}{l}1 \\
2\end{array}$ & $\begin{array}{c}41 \\
\text { Desconhecido }\end{array}$ & $\begin{array}{c}\text { Assintomático } \\
-\end{array}$ & Enzimas alteradas \\
\hline $\mathrm{SE}_{3}$ & $\begin{array}{l}1 \\
1 \\
1 \\
1 \\
1 \\
1 \\
1\end{array}$ & $\begin{array}{c}19 \\
68 \\
40 \\
5 \\
\text { Desconhecido } \\
\text { Desconhecido } \\
37\end{array}$ & $\begin{array}{c}\text { Assintomático } \\
\text { Assintomático } \\
\text { Assintomático } \\
\text { Insatisfatório } \\
- \\
- \\
\text { Assintomático }\end{array}$ & $\begin{array}{c}\text { Enzimas normais } \\
\text { Enzimas normais } \\
\text { Enzimas normais } \\
\text { Enzimas alteradas } \\
- \\
- \\
\text { RV }\end{array}$ \\
\hline $\mathrm{SE}_{4}$ & $\begin{array}{l}1 \\
1 \\
1 \\
1 \\
1 \\
1 \\
1 \\
\end{array}$ & $\begin{array}{c}67 \\
40 \\
\text { Desconhecido } \\
41 \\
120 \\
2 \\
\text { Desconhecido } \\
\end{array}$ & $\begin{array}{c}\text { Assintomático } \\
\text { Assintomático } \\
- \\
\text { Óbito } \\
\text { Insatisfatório } \\
\text { Assintomático } \\
\text { - } \\
\end{array}$ & $\begin{array}{c}\text { Enzimas normais } \\
\text { RV } \\
- \\
- \\
\text { Enzimas alteradas } \\
\text { RV }\end{array}$ \\
\hline Total & 28 & - & - & - \\
\hline
\end{tabular}




\section{DISCUSSÃO}




\subsection{Discussão}

Dentre as complicações que podem advir da realização da colecistectomia, certamente a que se reveste de grande importância - não apenas pela freqüência com que tem ocorrido, mas principalmente pela gravidade de suas conseqüências - é a lesão da via biliar principal (VBP) Cunha (1998). Estudo multicêntrico, realizado por Bismuth (1981), relata incidência de $0,2 \%$ de lesões iatrogênicas das vias biliares (VB) em colecistectomia por laparotomia.

A incidência de lesão das VB reduziu para aproximadamente 0 a $0,2 \%$, à medida que os cirurgiões tornaram-se mais experientes com a cirurgia aberta (BLUMGART, 1988; MOOSA et al., 1992). Estudos recentes estimam que a maioria das estenoses benignas é de natureza iatrogênica, podendo, portanto, ser evitadas (SCHIRMER et col., 1991).

No período estudado foram realizadas 1.361 colecistectomias no HC da UFG, das quais $87,5 \%$ indicadas como procedimento eletivo e $14,3 \%$ procedimento de urgência. Neste grupo de pacientes operados ocorreram 12 $(0,9 \%)$ lesões de VB. No grupo de pacientes oriundos de outras regiões ocorreram 19 lesões de VB, sendo 15 em caráter eletivo e 4 em caráter de urgência; no total, houve 31 ocorrências de lesões de VB tratadas no Serviço de Cirurgia do HC da UFG. Não houve variação significativa quanto à freqüência de lesões entre as operações realizadas eletivamente e as de urgência. 
Publicações recentes enfatizam o maior risco de lesão em processos inflamatórios agudos, gordura no hilo hepático, obesidade, anatomia variante, comprimento insuficiente da incisão e não realização da $\mathrm{ClO}$ (ROSSI et al., 1992; MOOSA et al., 1993). Talvez não se possam comparar casuísticas em condições sociais tão diversas. Por exemplo, Bergman et al (1996) analisaram a incidência de lesões em uma série em que a principal indicação para colecistectomia laparoscópica foi, literalmente, "cálculos biliares sintomáticos não complicados". Em nosso meio sabe-se que, ao chegar ao hospital público, vindo às vezes de outras cidades ou Estados, o paciente já apresentou várias crises de que remitiram; ou pode estar em uma crise aguda há vários dias, procurando atendimento; então tratam-se com freqüência, de doenças em estágios diferentes de fibrose e inflamação.

Nas colecistectomias difíceis, quando na maioria dos casos o cirurgião se depara com um bloco de aderências causado por processo inflamatório, identificar as estruturas da tríade portal é tarefa difícil. O grande omento e o cólon transverso, freqüentemente, encontram-se aderidos à borda do fígado; o duodeno está aderido ao colo da vesícula biliar e, em ambos os casos, precisam ser liberados (HESS, 1960; BOULVIN, 1962; CHODOFF, 1961; GALLAGHER, 1960). Foi observado, nesta casuística, apenas um caso.

A média de idade, no presente estudo, é mais alta que a observada por outros autores, cujos pacientes estavam em faixas de 30 a 40 anos (MERCADO et al., 2000; HART et al., 2000). Em nosso meio a média foi de 43 anos (PENTEADO, 2002). Esta observação reforça a idéia de que os 
pacientes aqui avaliados têm a doença biliar há mais tempo, em estágio mais avançado.

Nesta casuística houve preponderância do sexo feminino, embora sem significado estatístico. O sexo feminino correspondeu a mais de $80 \%$ das operações.

A incisão mais utilizada foi a transversa subcostal. A laparotomia mediana, usada em 8 casos - mais como laparotomia exploradora apresenta inconveniente quanto à dificuldade de se afastar a parede no sentido lateral (HESS, 1960). A incisão para-mediana adotada pela escola americana apresenta vantagem quanto à exposição do campo operatório. Mas, por não ser isenta de complicações, como as hérnias incisionais e eventrações, que evoluem com índice considerável - em torno de 5,6\% - dita incisão foi abandonada entre nós (GARCIA-VALDECASAS, 1988; ALI J et col., 1979). Passaram então a predominar as incisões transversais, por suas inúmeras vantagens: primeiro, porque ocorre a firmeza da reparação e a reduzida restrição respiratória no pós-operatório; segundo, porque permite uma boa exposição do campo operatório (GRANTCHAROV, 2001). A tendência atual é de se horizontalizar esta incisão. Nos casos favoráveis, recomenda-se a minilaparotomia horizontal, por melhor respeitar as estruturas da parede abdominal, com ênfase à estética (HESS, 1960). A minilaparotomia tornou-se a intervenção de escolha, para alguns autores, quando se realiza a colecistectomia pelo método convencional (DUBOIS, 1982; PREVOST, 1985; SALEMBIER, 1986). 
O traçado da incisão é feito antes da intervenção, em função da morfologia do paciente e de eventuais incisões anteriores.

Não foi observada, nesta casuística, associação entre o tipo de incisão e a lesão da via biliar.

No período pós-operatório, quase todos os casos com lesão de via biliar apresentaram icterícia (93\%), que é o sinal mais importante para alertar da necessidade de investigação. Peritonite (9\%), colangite $(25,8 \%)$, fístula cutânea $(25,8 \%)$ e sepse $(6,4 \%)$ foram menos freqüentes.

O diagnóstico precoce é importante porque a condição clínica pode rapidamente deteriorar em 3 a 5 dias, quando aparecem íleo, peritonite e sepse (BERGMAN et al., 1996). Vários autores têm enfatizado a importância de investigação diagnóstica em pacientes com dor abdominal difusa, febre, queda do estado geral ou alteração da função hepática após colecistectomia (BERGMAN et al., 1996; BRANUN et al., 1993).

$\mathrm{Na}$ investigação pós-operatória foram usados vários recursos diagnósticos. As colangiografias foram utilizadas em $51,8 \%$ dos casos. A colangiografia endoscópica foi a mais freqüentemente indicada, em $26 \%$ da casuística, porque pode ser também terapêutica.

A colangiografia pré-operatória (CPO) ou intra-operatória $(\mathrm{CIO})$ tem, por finalidade essencial, localizar o local exato da lesão ou da estenose da árvore biliar, permitindo ao cirurgião escolher a modalidade de tratamento a ser empregada (MOREAUX, 1993; BERGMAN et al., 1996; DAVID et al., 2003). 
A ClO através da canulação do ducto cístico permite avaliar o seu comprimento e reconhecer as eventuais anomalias dos ductos biliares. Ela representa o exame essencial para diagnosticar lesão da VBP em cirurgia aberta ou laparoscópica, desde que as chapas sejam de boa qualidade e que sejam interpretadas corretamente (ANDRÉN-SANDENBERG, 1985). A ClO permite, em $3 \%$ a $5 \%$ dos casos, identificar cálculos latentes na via biliar, ainda que esta se apresente com diâmetro reduzido - além de ter o mérito de confirmar a integridade da VB durante a colecistectomia.

A ClO constitui procedimento que permite reduzir a necessidade de exploração da VBP no pré ou no pós-operatório; reduz a incidência, bem como a gravidade de lesão e, além disso, os custos são menores ou iguais aos custos do tratamento das lesões da VBP (PHILLIPS, 1993).

A relação custo/benefício da $\mathrm{ClO}$ foi muito bem analisada, em nosso meio. Bresciani (1992) sugere que sejam incluídos, nestes custos, eventuais reintervenções, procedimento endoscópico e reoperações, que podem chegar até o transplante de fígado, e que propicia uma "economia" com o uso deste exame $(\mathrm{ClO})$.

Em vários casos foram feitos mais de um exame complementar. A combinação de exames permitiu avaliar o nível da lesão e planejar o tratamento.

A ultra-sonografia (USG) foi o exame empregado em 13 pacientes, mostrando dilatação da via biliar intra-hepática. Em 9 pacientes as tomografias computadorizadas possibilitaram identificar o nível da lesão. 
Estes exames de imagem puderam orientar o cirurgião quanto à conduta do tratamento (Anexo 1).

Para analisar resultados de um tratamento é necessário separar pacientes com gravidades diferentes. As lesões decorrentes de colecistectomia podem se manifestar numa fase precoce ou tardia. Uma lesão parcial se manifesta precocemente com fístula e coleperitôneo; outras podem evoluir assintomáticas e manifestam-se tardiamente como estenose cicatricial (CHAUDARY, 2001). Para estas, a classificação de Bismuth, de 1981, tem sido utilizada. Recentemente, com o advento da videolaparoscopia, várias publicações sobre lesão de via biliar apresentam tentativas de sistematização para orientar o tratamento (SCHOL et al., 1995; BERGMAN et al., 1996). No presente estudo adotamos a classificação proposta por Strasberg, em 1996, que, embora se refira a lesões ocorridas em acesso laparoscópico, é bastante abrangente, inclui lesões que se manifestam tanto precoce quanto tardiamente; e, nas lesões do tipo SE inclui as classificadas por Bismuth (1981).

Os pacientes foram classificados, retrospectivamente, de acordo com os achados de exames complementares e da cirurgia para correção da lesão. Observamos 5 (16,1 \%) lesões tipo SD e $3(9,7 \%)$ do tipo SB. As lesões parciais que forem identificadas e tratadas precocemente, ou ainda durante a operação, podem evoluir de maneira satisfatória.

Nesta casuística somente uma lesão foi identificada durante a operação $(3,2 \%)$. A identificação da lesão durante o procedimento que a originou é pouco freqüente. Por certo, temos aí a razão de haver poucas 
publicações enfocando como proceder no momento da ocorrência da lesão (BRANUM et al., 1993; ADAMS et al., 1993; DAVIDS et al., 1993).

Diversos estudos apresentam índices elevados de lesões iatrogênicas da VB que deixaram de ser reconhecidas por ocasião das operações. Destes estudos destacam-se: Lahey (1950) refere índice de até 50\%; Warren et col (1973) relata índice de 15\%; Longmire (1996) estima este índice em torno de 62,5\%; Pitt et col (1983) avaliaram este índice em 75\%; Bismuth (1983) relata índice de $45 \%$ e, Penteado (2000) estimou estes dados em $85 \%$.

A identificação mais precoce teria o mérito de interromper o curso das complicações. Entretanto, ao avaliar o trabalho, este fato não é observado. O tratamento mais precoce nem sempre correspondeu aos melhores resultados; em um caso refletiu a gravidade da lesão com transecção dos hepáticos direito e esquerdo. As lesões que causaram sinais e sintomas tardiamente - cuja reconstrução foi eletiva - apresentaram melhores resultados. Esta observação também foi confirmada por Bergman et al (1996).

Provavelmente algumas lesões menores vão se manifestar, como estenose cicatricial, tardiamente, após meses ou anos. Portanto, a reoperação para os estreitamentos das lesões constituiu uma necessidade, na maioria dos casos.

De acordo com a natureza das lesões e o quadro clínico, foi programado $\circ$ tratamento cirúrgico. A hepaticojejunoanastomose foi $\circ$ tratamento cirúrgico utilizado em $20(64, \%)$ pacientes; noutros dois $(6,4 \%)$, 
empregou-se a coledocoduodenoanastomose, porque eram casos de lesões distais.

Ao todo, em 9 (29\%) oportunidades, foram realizadas drenagens da via biliar: em $7(22,5 \%)$, com o objetivo de resolver uma lesão lateral, e em outros $2(6,4 \%)$ com o objetivo de resolver uma situação de urgência e preparar para o tratamento definitivo posterior. Esta conduta é aconselhada por Bergman et al (1996) que observaram mais complicações associadas às reconstruções de urgência que às eletivas.

Os drenos, principalmente tipo Kehr, permaneceram de 7 a 270 dias, de acordo com cada caso. Em uma paciente o dreno foi mantido até o final do período puerperal, 270 dias. Em dois casos de operações de urgência optou-se por drenagem através de sondas de Foley; estes evoluíram para o óbito, antes do tratamento definitivo.

A complicação pós-operatória mais freqüente, $29 \%$, é a infecção da ferida operatória. Fístulas biliares foram observadas em 5 casos (16\%).

Três pacientes faleceram devido a sepse. Todos apresentavam lesões altas, tipos $\mathrm{SE}_{3}$ e $\mathrm{SE}_{4}$. As doenças associadas não acarretaram mortalidade ao tratamento cirúrgico.

No que se refere ao seguimento tardio, tem-se que os pacientes com lesões tipo SB e SD, acompanhados de 9 a 40 meses, encontram-se assintomáticos. Outros pacientes apresentaram lesões mais complexas, tipo $\mathrm{SE}$, no seguimento de dois meses a doze anos. Estes em sua maioria, estão assintomáticos, com enzimas normais, também não necessitando de tratamento. Apenas três apresentaram enzimas alteradas; num, as enzimas 
alteradas eram pré-existentes à correção; noutro foi feito tratamento endoscópico. Um terceiro paciente também já apresentava cirrose biliar, mas não aceita retornar para exames.

Em suma, não foi possível, como seria desejável, estabelecer um padrão de circunstâncias associadas a um maior risco de lesão da via biliar. Por outro lado, ficou evidente que pacientes que apresentaram icterícia após a colecistectomia teriam que ser submetidos a investigação incisiva.

Como esta casuística compreendeu pacientes com lesões de gravidades diferentes, não foi possível comparar tratamentos. O sistema de classificação adotado pode ser considerado válido, porque separou adequadamente os casos.

As lesões parciais SB e SD evoluíram com melhores resultados no acompanhamento tardio de até 5 anos. Os óbitos corresponderam às lesões do tipo SE.

Com a experiência deste estudo será possível formular um protocolo de investigação e tratamento adequado para cada tipo de lesão. 


\section{CONCLUSÕES}




\subsection{Conclusões}

As lesões de via biliar ocorreram em $0,9 \%$ das colecistectomias realizadas no $\mathrm{HC}$ da UFG.

Não foi observada correlação entre lesão de via biliar e o fato de a colecistectomia ser eletiva ou de urgência $(p=0,161)$.

Não foi observada correlação entre lesão e sexos $(p=0,257)$.

A lesão mais freqüentemente observada é a secção proximal tipo $\mathrm{SE}_{4}$.

As lesões parciais dos ductos, tipos SB e SD apresentaram melhores resultados a longo prazo que as do tipo SE.

Os resultados dos exames complementares das lesões do tipo SB e SD são mostrados no Anexo 1. E os que se referem às lesões do tipo SE são mostradas no Anexo 2.

Sete pacientes necessitaram de dois procedimentos cirúrgicos (Anexo 3) e três pacientes foram submetidos a três intervenções para tratamento das lesões das vias biliares (Anexo 4).

Os óbitos se restringiram aos casos de lesão total proximal, $\mathrm{SE}_{3} \mathrm{e}$ $\mathrm{SE}_{4}$ 
ANEXOS 
ANEXO 1 - Achados dos exames complementares dos pacientes com LVB tipo SB e SD segundo a classificação de Strasberg

\begin{tabular}{|c|c|c|c|c|}
\hline $\begin{array}{c}\text { Tipo de } \\
\text { lesão }\end{array}$ & $\mathbf{n}$ & Exame & Achados & Tipo de cirurgia \\
\hline \multirow[t]{4}{*}{ SB } & 1 & & $\begin{array}{l}\text { dilatação VB intra-hepáticas } \\
\text { + bilioma }\end{array}$ & Drenagem de DHD \\
\hline & & USG Ab & $\begin{array}{l}\text { coleção sub-hepática + } \\
\text { abscesso hepático }\end{array}$ & \\
\hline & 1 & TC Ab & dilatação VB intra-hepáticas & Drenagem de DHD \\
\hline & $1^{*}$ & NA & & Drenagem de DHD \\
\hline \multirow[t]{6}{*}{ SD } & 1 & CPER & papilite (papilotomia) & Drenagem de DCD \\
\hline & & USG Ab & $\begin{array}{l}\text { ducto hepato-colédoco não } \\
\text { visualizado }\end{array}$ & \\
\hline & 1 & CPER & $\begin{array}{l}\text { papilite + dilatação de VB } \\
\text { extra-hepáticas (papilotomia) }\end{array}$ & Drenagem de DCD \\
\hline & 1 & TC Ab & dilatação VB intra-hepáticas & Drenagem de DCD \\
\hline & & USG Ab & coleção sub-hepática & \\
\hline & $2^{* *}$ & NA & & Drenagem de DCD \\
\hline
\end{tabular}

$\mathrm{n}=$ Número de pacientes

* Tratado no intra-operatório

** 1 paciente tratado no intra-operatório e 1 no 1 PO

$\mathrm{VB}=$ Vias biliares

NA = Não se aplica

$\mathrm{DCD}=$ Ducto colédoco

$\mathrm{DHD}=$ Ducto hepático direito 


\section{ANEXO 2 - Achados dos exames complementares dos pacientes com LVB tipo SE segundo a classificação de Strasberg}

\begin{tabular}{|c|c|c|c|c|}
\hline $\begin{array}{l}\text { Tipo de } \\
\text { lesão }\end{array}$ & $\mathrm{n}$ & Exame & Achados & Tipo de cirurgia \\
\hline \multirow[t]{2}{*}{$\mathrm{SE}_{1}$} & 1 & $\begin{array}{l}\text { TC Ab } \\
\text { USG Ab }\end{array}$ & $\begin{array}{l}\text { estenose do DCD + coledocolitíase } \\
\text { dilatação VB intra e extra-hepáticas + } \\
\text { coleção sub-hepática } \\
\text { normal } \\
\text { estenose (cordpo estranho) (papilotomia) }\end{array}$ & $\mathrm{HJ}$ \\
\hline & 1 & $\begin{array}{l}\text { USG Ab } \\
\text { CPER } \\
\text { USG Ab } \\
\text { TC Ab }\end{array}$ & $\begin{array}{l}\text { dilatação VB intra e extra-hepáticas } \\
\text { dilatação VB intra e extra-hepáticas + } \\
\text { estenose de }\end{array}$ & $\begin{array}{l}\text { CD } \\
\text { DCD }\end{array}$ \\
\hline $\mathrm{SE}_{2}$ & $\begin{array}{c}1 \\
2^{*}\end{array}$ & $\begin{array}{l}\text { CPER } \\
\text { NA }\end{array}$ & estenose de DCD (papilotomia) & $\begin{array}{l}\mathrm{HJ} \\
\mathrm{HJ}\end{array}$ \\
\hline \multirow[t]{2}{*}{$\mathrm{SE}_{3}$} & 1 & $\begin{array}{l}\text { CPER } \\
\text { TC Ab }\end{array}$ & $\begin{array}{l}\text { estenose de DHC / estenose de DCD } \\
\text { dilatação VB intra e extra-hepáticas + } \\
\text { cálculos intra-hepáticos + estenose de DCD } \\
\text { hepatomegalia }\end{array}$ & $\begin{array}{l}\mathrm{HJ} \\
\mathrm{HJ}\end{array}$ \\
\hline & 1 & $\begin{array}{l}\text { USG Ab } \\
\text { USG Ab } \\
\text { CPER } \\
\text { TC Ab } \\
\text { TPH } \\
\text { CPER } \\
\text { USG Ab } \\
\text { TPH }\end{array}$ & $\begin{array}{l}\text { hepatomegalia } \\
\text { dilatação de VB intra-hepáticas } \\
\text { estenose de DCD } \\
\text { dilatação de VB intra-hepáticas + bilioma } \\
\text { estenose de DHCo } \\
\text { estenose de DCD } \\
\text { dilatação VB intra e extra-hepáticas } \\
\text { dilatação VB intra e extra-hepáticas + } \\
\text { drenagem externa }\end{array}$ & $\begin{array}{l}\text { HJ } \\
\text { HJ }\end{array}$ \\
\hline \multirow[t]{2}{*}{$\mathrm{SE}_{4}$} & $\begin{array}{l}2 \\
4 \\
1\end{array}$ & $\begin{array}{l}\text { NA } \\
\text { USG Ab } \\
\text { USG Ab } \\
\text { CR } \\
\text { USG Ab }\end{array}$ & $\begin{array}{l}\text { estenose em nível de DHC } \\
\text { dilatação VB intra e extra-hepáticas } \\
\text { estenose de DCD proximal } \\
\text { coleccão sub-hepática }\end{array}$ & $\begin{array}{l}\mathrm{HJ} \\
\mathrm{HJ}+1 \text { Drenagem } \\
\mathrm{HJ}\end{array}$ \\
\hline & $\begin{array}{l}1 \\
1 \\
1\end{array}$ & $\begin{array}{l}\text { CPER } \\
\text { CPER } \\
\text { TC Ab } \\
\text { USG Ab } \\
\text { TC Ab }\end{array}$ & $\begin{array}{l}\text { estenose de DHC proximal } \\
\text { estenose de DCD } \\
\text { dilatação VB intra e extra-hepáticas } \\
\text { dilatação VB intra e extra-hepáticas } \\
\text { dilatação VB intra e extra-hepáticas }\end{array}$ & $\begin{array}{l}\mathrm{HJ} \\
\mathrm{HJ} \\
\text { Drenagem }\end{array}$ \\
\hline \multicolumn{5}{|c|}{$\begin{array}{l}\mathrm{n}=\text { Número de pacientes } \\
* 1 \text { paciente abdome agudo no } 6^{\circ} \mathrm{PO} \text { e } 1 \text { paciente com fístula biliar no } \mathrm{PO}\end{array}$} \\
\hline \multicolumn{5}{|c|}{$\begin{array}{l}\text { DHC = Ducto hepato-colédoco } \\
\text { DHCo = Ducto hepático comum } \\
\text { DCD = Ducto colédoco } \\
\text { VB = Vias biliares }\end{array}$} \\
\hline
\end{tabular}




\section{ANEXO 3 - Intervalo entre a colecistectomia e as cirurgias corretivas nos pacientes que necessitaram de 2 procedimentos}

\begin{tabular}{|c|c|c|c|c|c|c|c|}
\hline Pacientes & $\begin{array}{l}\text { Intervalo } \\
\text { de tempo } \\
\text { (dias) }\end{array}$ & $\begin{array}{c}\text { Tipo de } \\
\text { lesão }\end{array}$ & 10 procedimento & $\begin{array}{l}\text { Intervalo } \\
\text { de tempo } \\
\text { (dias) }\end{array}$ & Complicaçōes & $2^{\circ}$ procedimento & $\begin{array}{l}\text { Complicaçōes após } \\
\text { tratamento definitivo }\end{array}$ \\
\hline MNFA & 63 & $\mathrm{SE}_{3}$ & Kehr no hepático $E$ & 300 & $\begin{array}{l}\text { Colangite de repetição, } \\
\text { empiema pleural após } \\
\text { TPH, fístula } \\
\text { hepaticoduodenal }\end{array}$ & HJ & $\begin{array}{l}\text { Hematoma } \\
\text { intracavitário } \\
\text { fistula cutânea }\end{array}$ \\
\hline RSB & 23 & $\mathrm{SE}_{1}$ & $\begin{array}{l}\text { CPER } \\
\text { Papilotomia - retirada } \\
\text { de cálculos }\end{array}$ & 11 & Icterícia, prurido & $C D$ & $\begin{array}{l}\text { Infecção de } \\
\text { ferida } \\
\text { operatória, } \\
\text { febre, diarréia } \\
\text { prolongada }\end{array}$ \\
\hline MAS & 53 & $\mathrm{SE}_{4}$ & HJ & 51 & $\begin{array}{l}\text { Estenose da } \\
\text { anastomose após } \\
10 \text { anos }\end{array}$ & $\begin{array}{l}\text { Dilatação com balão, } \\
\text { cortes axiais com } \\
\text { papilotomo } \\
\text { Reposicionada alça à } \\
\text { Voelcker }\end{array}$ & - \\
\hline BPS & 5 & SD & $\begin{array}{l}\text { Coledocorrafia + } \\
\text { coledocostomia com } \\
\text { Kehr }\end{array}$ & 48 & $\begin{array}{l}\text { Infecção da ferida } \\
\text { operatória, colangite, } \\
\text { papilite }\end{array}$ & CPER + Papilotomia & - \\
\hline AMAL & 10 & $\mathrm{SE}_{3}$ & $\begin{array}{l}\text { Coledocostomia } \\
\text { com Kehr }\end{array}$ & 34 & $\begin{array}{l}\text { Icterícia persistente, } \\
\text { Diarréia }\end{array}$ & HJ & - \\
\hline SMJ & 147 & $\mathrm{SE}_{4}$ & HJ & 613 & Icterícia, colangite & HJ & - \\
\hline MAF & 63 & $\mathrm{SE}_{4}$ & $\begin{array}{l}\text { Coledocostomia } \\
\text { com Kehr }\end{array}$ & 392 & $\begin{array}{l}\text { Cirrose biliar } \\
\text { secundária, } \\
\text { fístula } \\
\text { coledocoduodenal, } \\
\text { fístula cutânea }\end{array}$ & HJ & - \\
\hline
\end{tabular}

$\mathrm{CD}=$ Coledocoduodenostomia

$\mathrm{HJ}=$ Hepaticojejunostomia 
ANEXO 4 - Intervalo entre a colecistectomia e as cirurgias corretivas nos pacientes que necessitaram de 3 procedimentos

\begin{tabular}{|c|c|c|c|c|c|c|c|c|c|c|}
\hline Pacien-tes & $\begin{array}{l}\text { Tipo de } \\
\text { lesão }\end{array}$ & $\begin{array}{l}\text { Intervalo } \\
\text { de tempo } \\
\text { (dias) }\end{array}$ & $1^{\circ}$ procedimento & Complicaçōes & $\begin{array}{l}\text { tervalo do tc } \\
\text { proce- } \\
\text { dimento } \\
\text { (dias) }\end{array}$ & $2^{\circ}$ procedimento & Complicaçôes & $\begin{array}{l}\text { Intervalo } \\
\text { do } 2^{0} \\
\text { proce- } \\
\text { dimento } \\
\text { (dias) }\end{array}$ & $\begin{array}{l}3^{\circ} \\
\text { proce- } \\
\text { dimento }\end{array}$ & Complicaçốes \\
\hline DCO & $\mathrm{SE}_{1}$ & 36 & $\begin{array}{l}\text { Papilotomia, } \\
\text { retirada de } \\
\text { corpo } \\
\text { estranho }\end{array}$ & $\begin{array}{l}\text { Dore } \\
\text { distensão } \\
\text { abdominal }\end{array}$ & 330 & $\begin{array}{l}\text { Coledocos- } \\
\text { tomia com } \\
\text { Kehr }\end{array}$ & $\begin{array}{l}\text { Estenose de } \\
\text { colédoco } \\
\text { distal, icterícia, } \\
\text { colúria, acolia } \\
\text { fecal }\end{array}$ & 123 & $C D$ & $\begin{array}{l}\text { Infecção } \\
\text { de ferida } \\
\text { operatória }\end{array}$ \\
\hline MGM & $\mathrm{SE}_{4}$ & 10 & $\begin{array}{l}\text { Colecistectom } \\
\text { ia, rafia de } \\
\text { DHD, } \\
\text { Coledocosto } \\
\text { mia com Kehr }\end{array}$ & $\begin{array}{l}\text { Hénia } \\
\text { incisional, } \\
\text { fístula } \\
\text { cutânea, } \\
\text { colangite, } \\
\text { icterícia }\end{array}$ & 370 & $\begin{array}{l}\text { CPER, } \\
\text { Tratamento } \\
\text { conservador }\end{array}$ & $\begin{array}{l}\text { Estenose de } \\
\text { colédoco } \\
\text { distal, } \\
\text { Hérnia } \\
\text { incisional }\end{array}$ & 98 & HJ & - \\
\hline MPPS & $\mathrm{SE}_{4}$ & 9 & $\begin{array}{l}\text { Drenagem } \\
\text { dos ductos } \\
\text { hepáticos SD } \\
\text { e SE com } \\
\text { Kher }\end{array}$ & $\begin{array}{l}\text { Dor } \\
\text { abdominal, } \\
\text { fístula } \\
\text { cutânea, } \\
\text { icterícia, } \\
\text { peritonite } \\
\text { biliar }\end{array}$ & 29 & $\begin{array}{l}\text { Drenagem } \\
\text { dos ductos } \\
\text { hepáticos SD } \\
\text { e SE com } \\
\text { Foley }\end{array}$ & $\begin{array}{l}\text { Peritonite biliar, } \\
\text { dor abdominal, } \\
\text { fístula interna }\end{array}$ & 59 & HJ & - \\
\hline
\end{tabular}

$\mathrm{CD}=$ Coledocoduodenostomia

$\mathrm{HJ}=$ Hepaticojejunostomia

$\mathrm{CPER}=$ Colangiopancreatografia endoscópica retrógrada 
ANEXO 5 - Acompanhamento tardio

\begin{tabular}{|c|c|c|c|c|c|c|}
\hline № & Strasberg & $\mathrm{n}$ & $\begin{array}{l}\text { Período } \\
\text { (meses) }\end{array}$ & Condição & & ames \\
\hline 1 & SB & 1 & 9 & Insatatório & RV & - \\
\hline 2 & SD & 1 & 31 & Satisfatório & $\begin{array}{l}\text { BT } \\
\text { BD } \\
\text { BI } \\
\text { TGO } \\
\text { TGP } \\
\text { G-GT } \\
\text { U S G }\end{array}$ & $\begin{array}{l}=0,6 \mathrm{mg} / \mathrm{dl} \\
=0,3 \mathrm{mg} / \mathrm{dl} \\
=0,3 \mathrm{mg} / \mathrm{dl} \\
=17,0 \mathrm{UI} / \mathrm{I} \\
=11,0 \mathrm{UI} / \mathrm{l} \\
=31,0 \mathrm{UI} / 1 \\
=\text { Normal }\end{array}$ \\
\hline 3 & SE1 & 1 & 48 & Satisfatório & $\begin{array}{l}\text { BT } \\
\text { Bd } \\
\text { BI } \\
\text { TGO } \\
\text { TGP } \\
\text { F.ALC }\end{array}$ & $\begin{array}{l}=0,6 \mathrm{mg} / \mathrm{dl} \\
=0,4 \mathrm{mg} / \mathrm{dl} \\
=0,2 \mathrm{mg} / \mathrm{dl} \\
=24,0 \mathrm{Ul} / \mathrm{l} \\
=13,6 \mathrm{UI} / / \\
=23,0 \mathrm{U} / \mathrm{l}\end{array}$ \\
\hline 4 & SD & 1 & 37 & Satisfatório & $\begin{array}{l}\text { BT } \\
\text { BD } \\
\text { BI } \\
\text { TGO } \\
\text { TGP } \\
\text { G-GT }\end{array}$ & $\begin{array}{l}=0,5 \mathrm{mg} / \mathrm{dl} \\
=0,3 \mathrm{mg} / \mathrm{dl} \\
=0,2 \mathrm{mg} / \mathrm{dl} \\
=22,0 \mathrm{UI} / / \\
=13,0 \mathrm{UI} / 1 \\
=14,0 \mathrm{U} / \mathrm{l}\end{array}$ \\
\hline 5 & $\mathrm{SE}_{3}$ & 1 & 07 & Óbito & - & - \\
\hline 6 & $\mathrm{SE}_{2}$ & 1 & & Ignorado & - & - \\
\hline 7 & SD & 1 & & Ignorado & - & - \\
\hline 8 & $\mathrm{SE}_{2}$ & 1 & 41 & Satisfatório & $\begin{array}{l}\text { TGO } \\
\text { TGP } \\
\text { G-GT } \\
\text { BT } \\
\text { BD } \\
\text { BI } \\
\text { USG }\end{array}$ & $\begin{array}{l}=186,0 \mathrm{Ul} / \mathrm{I} \\
=141,0 \mathrm{UI} / \mathrm{l} \\
=949,0 \mathrm{U} / \mathrm{l} \\
=2,7 \mathrm{mg} / \mathrm{dl} \\
=1,3 \mathrm{mg} / \mathrm{dl} \\
=1,4 \mathrm{mg} / \mathrm{dl} \\
=\text { Normal }\end{array}$ \\
\hline 9 & SB & 1 & 30 & Satisfatório & $\mathrm{RV}$ & - \\
\hline 10 & $\mathrm{SE}_{4}$ & 1 & 40 & Satisfatório & RV & - \\
\hline 11 & $\mathrm{SE}_{4}$ & 1 & 55 & Satisfatório & $\begin{array}{l}\text { BT } \\
\text { BD } \\
\text { BI } \\
\text { TGO } \\
\text { TGP } \\
\text { G-GT } \\
\text { USG }\end{array}$ & $\begin{array}{l}=0,80 \mathrm{mg} / \mathrm{dl} \\
=0,40 \mathrm{mg} / \mathrm{dl} \\
=0,40 \mathrm{mg} / \mathrm{dl} \\
=32,0 \mathrm{UI} / 1 \\
=26,0 \mathrm{UI} / \mathrm{l} \\
=25,0 \mathrm{U} / \mathrm{l} \\
=\text { Normal }\end{array}$ \\
\hline 12 & SD & 1 & 40 & Satisfatório & RV & - \\
\hline 13 & $\mathrm{SE}_{3}$ & 1 & 8 & Satisfatório & $\begin{array}{l}\text { BT } \\
\text { TGP } \\
\text { G-GT } \\
\text { F. Alc }\end{array}$ & $\begin{aligned} & \ldots \\
= & 111,0 \mathrm{UI} / \mathrm{I} \\
= & 150,0 \mathrm{U} / \mathrm{l} \\
= & 341,0 \mathrm{U} / \mathrm{l}\end{aligned}$ \\
\hline 14 & SD & 1 & & Ignorado & - & - \\
\hline 15 & $\mathrm{SE}_{4}$ & 1 & 2 & Satisfatório & RV & - \\
\hline 16 & $\mathrm{SE}_{4}$ & 1 & 14 & Insatisfatório & $\begin{array}{l}\text { BT } \\
\text { BD } \\
\text { BI } \\
\text { TGO } \\
\text { TGP } \\
\text { AMIL } \\
\text { F.Alc. } \\
\text { G-GT }\end{array}$ & $\begin{array}{r}=1,2 \mathrm{mg} / \mathrm{dl} \\
=0,4 \mathrm{mg} / \mathrm{dl} \\
=0,8 \mathrm{mg} / \mathrm{dl} \\
=4 \mathrm{U} / \\
=53 \mathrm{U} / \mathrm{l} \\
=122 \mathrm{un} / \mathrm{dl} \\
=157 \mathrm{U} / 1 \\
=162,0 \mathrm{UI} / 1\end{array}$ \\
\hline
\end{tabular}


conclusão

ANEXO 5 - Acompanhamento tardio

\begin{tabular}{|c|c|c|c|c|c|c|}
\hline № & Strasberg & $\mathrm{n}$ & $\begin{array}{l}\text { Período } \\
\text { (meses) }\end{array}$ & Condição & \multicolumn{2}{|c|}{ Exames } \\
\hline 17 & $\mathrm{SE}_{1}$ & 1 & 60 & Satisfatório & $\begin{array}{l}\text { USG } \\
\text { BT } \\
\text { BD } \\
\text { BI } \\
\text { G-GT }\end{array}$ & $\begin{aligned} & =\text { Normal } \\
= & 0,4 \mathrm{mg} / \mathrm{dl} \\
= & 0,2 \mathrm{mg} / \mathrm{dl} \\
= & 0,2 \mathrm{mg} / \mathrm{dl} \\
= & 17,5 \mathrm{UI} /\end{aligned}$ \\
\hline 18 & $\mathrm{SE}_{1}$ & 1 & 32 & Satisfatório & RV & - \\
\hline 19 & $\mathrm{SE}_{3}$ & 1 & & Ignorado & & - \\
\hline 20 & $\mathrm{SE}_{3}$ & 1 & 36 & Satisfatório & RV & - \\
\hline 21 & $\mathrm{SE}_{4}$ & 1 & 1 & Óbito & - & - \\
\hline 22 & $\mathrm{SE}_{4}$ & 1 & 40 & Ignorado & - & - \\
\hline 23 & $\mathrm{SE}_{3}$ & 1 & & Ignorado & - & - \\
\hline 24 & SB & 1 & & Ignorado & - & - \\
\hline 25 & $\mathrm{SE}_{2}$ & 1 & & Ignorado & - & - \\
\hline 26 & $\mathrm{SE}_{3}$ & 1 & 19 & Satisfatório & $\begin{array}{l}\text { BT } \\
\text { BD } \\
\text { BI } \\
\text { TGO } \\
\text { TGP }\end{array}$ & $\begin{array}{l}=0,10 \mathrm{mg} / \mathrm{dl} \\
=0,06 \mathrm{mg} / \mathrm{dl} \\
=0,04 \mathrm{mg} / \mathrm{dl} \\
=37,0 \mathrm{UI} / 1 \\
=47,0 \mathrm{UI} / 1\end{array}$ \\
\hline 27 & $\mathrm{SE}_{4}$ & 1 & & Ignorado & - & - \\
\hline 28 & $\mathrm{SE}_{3}$ & 1 & 69 & Satisfatório & $\begin{array}{l}\text { BT } \\
\text { BD } \\
\text { BI } \\
\text { TGO } \\
\text { TGP }\end{array}$ & $\begin{array}{l}=0,80 \mathrm{mg} / \mathrm{dl} \\
=0,40 \mathrm{mg} / \mathrm{dl} \\
=0,40 \mathrm{mg} / \mathrm{dl} \\
=18,0 \mathrm{UI} / 1 \\
=20,0 \mathrm{UI} / 1\end{array}$ \\
\hline 29 & $\mathrm{SE}_{4}$ & 1 & 48 & Óbito & - & - \\
\hline 30 & $\mathrm{SE}_{3}$ & 1 & 40 & Satisfatório & USG & $=$ Normal \\
\hline 31 & $\mathrm{SE}_{4}$ & 1 & 49 & Óbito & & \\
\hline
\end{tabular}




\section{REFERÊNCIAS BIBLIOGRÁFICAS}




\subsection{Referências Bibliográficas}

ADAMS DB, BOROVICZ MR, WOOTTON FT, CUNNINGHAM JT. Bile duct complicatios after laparoscopic cholecystectomy. Surg Endosc, 7: 79-83, 1993.

ALI J, ALI KHAN T. The comparative effects of muscle transection and median upper abdominal incisions on postoperative pulmonary function. Surg Gynecol Obstet, 148: 863-6, 1979.

ANDRÉN-SANDENBERG G, BENGMAR K. Accidental Lesions of the Common Bile Duct at Cholecystectomy. Pre and Perioperative factors of importance. Ann Surg, 201: 328-32, 1985.

ATTILI AF, CARULLI N, RODA E. Epidemiology of gallstone disease in Italy: prevalence date of the multicenter Italian study on cholelithiasis. Am $\mathbf{J}$ Epidemiol, 141: 158-65, 1995.

BARBARA L, SAMA C, MORSELI LABATE AP et col. A population study on the prevalence of gallstone disease: The Sirmione study. Hepatology, 7: 91317, 1987.

BERGMAN JJHGM, van den BRINK GR, RAWS EAJ, de WIT, OBERTOP H, HUIBREGTSE K, TYTGAT GNJ, GOUMA DJ. Treatment of bile duct lesion after laparoscopic cholecystectomy. Gut, 38:141-7, 1996.

BIRINDELLI JP. Complicações da cirurgia biliopancreática. In: Speranzini M, Oliveira MR. Manual do residente de cirurgia. Rio de Janeiro, GuanabaraKoogan, 88: 508-14, 1988. 
BISMUTH H, LAZORTHES $F$. Les traumatismes opératoires de la voie biliaire principale. Rapport au $83^{\grave{e}}$ Congrès de Chirurgie. Monografie de IAFC. Masson Ed (Paris), 1: 1981.

BISMUTH H, PIETRO EM. Biliary Strictures: Classification based on the principles of surgical treatment. World J Surg, 25: 1241-4, 2001.

BISMUTH H. Postoperative strictures of the biliary tract. In Blumgart LH (ed): The Biliary Tract. Clinical Surgery International. Edinburgh Churchill Livingstone, 5: 209-18, 1983.

BISS K, HO KJ, MIKKELSON B, LEWIS L, TAYLOR CB. Some unique biologic characteristics of the Massai of East Africa. N Engl J Med, 284: 694-8, 1971.

BLUMGART LH. Benign biliary strictures. Blumgart LH, 721-52, 1988.

BOUCHIER IAD. Gallstones: formation and epidemiology. In: Blumgart LH ed. Surgery of the liver and the Biliary Tract. Edinburgh: Churchill Livingstone, 503-16, 1988.

BOULVIN R. 125 cas de cholecystites aiguës. Ann Chir (Paris), 16:1249, 1962.

BRANUM G, SCHMITT C, BAILLIE $J$ et al. Management of major biliary complications after laparoscopic cholecystectomy. Ann Surg, 217 : 532$41,1993$.

BRESCIANI C, GAMA-RODRIGUES J et al. Colangiografia intra-operatória em vídeo-laparoscopia. Experiência em 157 casos. In: Bresciani C, GamaRodrigues j, Habr-Gama A, Carrilho FJ, Lech J. ed. Cirurgia vídeolaparoscópica e laparoscopia diagnóstica em aparelho digestivo. São Paulo, Editora Independente, pp 200-2, 1992. 
BRESCIANI C, GAMA-RODRIGUES J, SATO NH et al. Colangiografia intraoperatória: o que ela acrescenta de custos e tempo à colecistectomia laparoscópica. In Habr-Gama A, Gama-Rodrigues J, Ceconello I, Zilberstein B, Machado MCC, Saad WA, Moura EGH, Bresciani C. ed. Atualização em cirurgia do aparelho digestivo e em coloproctologia. São Paulo, Frôntis Editorial, pp 83-9, 2000.

CHAPMAN BA, WILSON IR, FRAMPTON CM et al. Prevalence of gallbladder disease in diabetes mellitus. Dig Dis Sci, 41: 222-8, 1996.

CHAUDHARY A, MANISEGRAN M, CHANDRA A, AGARWAL A K, SACHDEV AK. How do bile duct injuries sustained during laparoscopic cholecystectomy differ from those during open cholecystectomy. J Laparoendosc Adv Surg Tech, 11: 187-91, 2001.

CHODOFF R. Surgical management of acute cholecystites. Amer J Gastroenterol, 109:60, 1961.

COELHO JCU, BONILHA R, PITAKI SAM et al. Prevalence of gallstones in a brazilian population. Int Surg, 84:25-8, 1999.

COLLET D, CROZAT T, ALHI S. Incidents et complications de la Cholécystectomie Coelioscopique. L'enquête de la SFCERO. Lyon Chir, 87: 463-6, 1991.

COLLINS PG, GOREY TF. latrogenic biliary stricture: presentation and management. Br J Surg, 71: 980-82,1984.

CUNHA JEM, ABDO EE, MACHADO MCC et al. Surgical treatment of cicatricial biliary strictures. Hepato- Gastroenterology, 45: 1452-56, 1998. 
CUSCHIERI A, DUBOIS F, MOURIEL J. et al. The European experience with Laparoscopic Cholecystectomy. Am J Surg, 161: 385-7, 1991.

DAVID R, DELLINGER E et al. Cholangiography and risk of common bile duct injury during cholecystectomy. JAMA, 289:1639-44, 2003.

DAVIDS PHP, RINGERS J, RAWS EAJ, de WIT LT, HUIGBRETSE Ket al. Bile duct injury after laparoscopic cholecystectomy: the value of endoscopic retrograde cholangiopancreatography. Gut, 34:1250-4, 1993.

DEZIEL DJ, MILLIKAN KW, ECONOMOU SG et al. Complications of Laparoscopic Cholecystectomy: A National survey of 4,292 hospitals and an analysis of 77,604 cases. Am J Surg, 165: 9-14, 1993.

DUBOIS F, BERTHELOT G. Cholécystectomie par minilaparotomie. Nouv Presse Med, 11: 1139-41, 1982.

DUBOIS F, ICARD P, BERTHELOT $G$ et al. Coelioscopic Cholecystectomy: Preliminary report of 36 cases. Ann Surg, 211: 60-2, 1990.

FRIEDMAN GD, KANNEL WB, DAWBER TR. The epidemiology of gallblader disease: observations in the framinghan study. J Chir Dis, 19: 273-92. 1966.

GALLAGHER WB. Perforation of the gallblader. Amer J Surg, 100: 407, 1960.

GARCIA-VALDECASAS JC, ALMENARA R, CABRERA C, DE LACY AM, SUST M, TAURA $P$ et al. Subcostal incision versus midline laparotomy in gallstones surgery; a prospective and randomized trial. Br J Surg, 75: 473-5, 1988.

GARDEN OJ. latrogenic injury to the bile duct. Br J Surg, 78:1412-3, 1991. 
GRACE PA, QURESHI A, BURKE P, LEAHY A, BRINDLEY N, OSBORNE H, LANE B, BROE P, BOUCHIER-HAYES D. Selective cholangiography in laparoscopic cholecystectomy. Br J Surg, 80: 244-6, 1993.

GRANTCHAROV TP et al. Vertical compared with transverse incision in abdominal surgery. Eur J Surg, 167:260-7, 2001.

HART RS, PASSSI RB, WALL WJ. Long-term outcome after repair of major bile duct injury created during laparoscopic cholecystectomy HPB, 2: 325-32, 2000.

HEATON KW. The epidemiology of gallstones and suggested etiology. Clin Gastroenterol; 2: 67-83, 1973.

HENRY ML, CAREY LC. Complications of cholecystectomy. Surg Clin North Am, 63: 1191-204, 1983..

HESS W. Surgery of the Biliary Passages and the Pancreas. Operative technique. D Van Nostrand Company Inc, 330-2, 1960.

HOFFMANN J, LORENTZEN M. Drainage after cholecystectomy. Br J Surg, 72:423-7, 1985.

KEHR H. Die Praxis der Gallenwege-chirurgie in Wort und Bild. München Lehmann, 1913.

LAHEY FH, PYRTEK LJ. Experience with operative management of 280 strictures of the bile ducts. Surg Gynec Obstet, 91: 25-6, 1950.

LANGENBUCH CJA. Ein Fall von Extirpation der Gallenbrase wegwn Chronisher Cholelitiasis: Heilung Berl Klin wochenchr, 19: 725-7, 1882. 
LEVY VG. La lithiase vésiculaire est-elle une maladie? Presse Med, 17: 1515-7, 1988.

LIGIDAKIS NJ. Operative risk factors of cholecystectomy-choledochotomy in the elderly. Surg Gynecol Obstet, 157: 15-9, 1983.

LONGMIRE WP. Early management of injury to the extrahepatic biliary tract. J Am Med Ass, 195: 623-5, 1966.

MACHI J, TATEISHI T, OISHI AJ, FURUMOTO NL, UCHIDA S, SINGEL B. Laparoscopic ultrasonography versus operative cholangiography during laparoscopic cholecystectomy. Review of the literature and comparison with open intraoperative ultrasonography. J Am Coll Surg, 188: 361-7, 1999.

MAYO WJ. Some remarks on cases involving operative loss of continuity of common bile duct with a report of cases of anastomosis between the hepatic duct and the duodenum. Ann Surg, 42: 90-6, 1905.

MC SCHERRY CK, GLENN F. The incidence and causes of death following surgery for nonmalignant biliary tract disease. Ann Surg, 191: 271, 1980.

MERCADO MA, OROZCO H, LOPEZ-MARTNEZ LM, PEREZ DEL VILLAR AP, TIELVE M, MARTINEZ R. Survival and quality of life after bile duct reconstruction for iatrogenic injury. HPB, 2: 321-4, 2000.

METMAN M. Morbidity de la lithiase biliaire. Symposium International lithiase biliaire et ursothérapie. Rev Fr Gastro-Enterol, 166: 15-6, 1981.

MONASTYRSKI ND, TILLING G. (Trans); Zur Fraje von der Chirurgischea Behandlung der vollständigen Undurchgängigket des Ductus Choledochus. Zentral bl Chir,15: 778-9, 1888. 
MONSON JR, GUILLOU PJ, KEANE FB et al. Cholecystectomy is safer without drainage: the results of a prospective, randomized clinical trial. Surgery; 109: 740-6, 1991.

MOOSA AR, EASTER DW, VON SANNENBERG E. Laparoscopic injuries to the bile duct. A cause for concern. Ann Surg, 215: 203-8, 1992.

MOREAUX J. Traitement des Complications de la Cholécystectomie. Éditions Techniques - Encycl Méd Chir. (Paris-France), Techniques Chirurgicales- Généralités-Appareil digestif, 18: 40-960, 1993.

MÜHE E. Die erste Cholecystektomie durch das Laparoscop. English Summary. Langenbecks Arch Klin Chir, 369: 804, 1986.

NOGUEIRA MVF, WINKLER A. Colangiografia transoperatória. Indicações e métodos. In: Creuz O. ed. Cirurgia video-endoscópica, São Paulo, Revinter, 151-8, 1993.

OLSEN DO. Laparoscopic cholecystectomy. Am J Surg, 161: 339-44, 1991.

PENTEADO S, CUNHA JEM, MACHADO MCC, et al. Tratamento Cirúrgico das Estenoses Cicatriciais. Em Atualização em cirurgia do aparelho digestivo e coloproctologia. Habr-Gama A, Gama-Rodrigues J, Machado MMC et al. Frôntis editorial S. Paulo, 237-40, 2002.

PERISSAT J, COLLET D, BELLIARD R et al. Laparoscopic cholecystectomy: The state of the art. A report on 700 consecutive cases. World J Surg, 16: 1074-82, 1992.

PHILLIPS EH. Routine versus selective intraoperative cholangiography. Am J Surg, 165: 505-7, 1993. 
PINZELLO R, MONGELLI D, CUSTODERO O, CATUCCI V, LEFEMINE S. Complicanze intra e postoperatorie nel trattamento chirurgico della colelitiase. G Chir, 11: 229-30, 1990.

PITT HA, MIYAMOTO T, PARAPATIS SK. et al: Factors influencing outocome in patients with postoperative biliary strictures. Am J Surg, 144: 14-21, 1982.

PITT HA, CAMERON JL, POSTIER RG. Et al. Factors affecting mortality in biliary tract surgery. Am J Surg, 141: 66-72, 1981.

PREVOST F, ROUSSET JF, FOURTANIER G, ESCAT J. Laparotomie horizontale en chirurgie biliaire. Presse Med, 14: 2059-60, 1985.

REDDICK EJ, OLSEN DO, SPAW A et al. Safe performance of difficult laparoscopic cholecystectomies. Am J Surg, 161: 377-81, 1991.

ROSENQUIST H, MYRIN SO. Operative injury to the bile ducts. Acta Chir Scand, 119: 92-107, 1996.

ROSSI RL, SCHIRMER WJ et al. Laparoscopic bile duct injuries. Arch surg, 127: 596-602, 1992.

SALEMBIER Y. Cholécystectomie par une courte incision transversale. Presse Med, 15: 210-11, 1986.

SAMPLINER RE, BENNETT PH, CONNESS LJ. Gallblader disease in Pima Indians. Demonstration of high prevalence and early onset by cholecystography. N Engl J Med; 283: 1358-64, 1970. 
SCHIRMER WJ, ROSSI RL, HUGHES KS, MUNSON L, BRAASCH JW. Common operative problems in hepatobiliary surgery. Surg Clin North Am, 71: 1363-89, 1991.

SCHOL FPG, GO PMNYH, GOUMA DJ. Autcome of 49 repair of bile duct injuries after laparoscopic cholecystectomy. World J Surg, 19: 753-7, 1995.

SHINAGAWA N, MASHITA K, YURA J. The operative risk factors of cholelithiasis in the elderly. Surg Today, 22: 34-9, 1992.

STRASBERG SM, CALLERY M.P, SOPER NJ. Laparoscopic Surgery of the bile ducts. Gastrointestinal Endoscopy. Clinics of North America, 6: 81-105, 1996.

SULLIVAN DM. Biliary tract surgery in the elderly. Am J Surg, 143:218, 1972.

TORVIK A, HÖIVIK B. Gallstones in an autopsy series. Acta Chir Scand, 120: 168-74, 1960.

TREROTOLA SO, SAVADER SJ, LUND GB, et al. Bile tract complications following laparoscopic cholecystectomy: imaging and intervention. Radiology, 184: 195-200,1992.

VON WINIWATER A, BIDDLER A (Trans). Ein Fall von Gallenretention bedingt durch impermeabilität des Ductus Choledochus. Anlegung einer Gallenblasen-Darmfistel: Heilung Zentralbe Chir, 9: 581-2, 1881.

WARREN KW, JEFFERSON MF. Prevention and repair of strictures of extrahepatic bile ducts. Surg Clin North Am 53: 1169- 90, 1973. 